\title{
ASSESSMENT OF FACTORS DETERMINING EFFICACY OF CARDIAC RESYNCHRONISATION THERAPY FOR PATIENTS WITH HEART FAILURE IN THE POPULATION OF LATVIA
}

Summary of the Doctoral Thesis for obtaining the degree of a Doctor of Medicine Specialty - Internal Medicine, Cardiology 


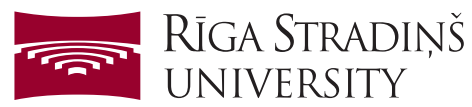

Maija Vikmane

\section{ASSESSMENT OF FACTORS \\ DETERMINING EFFICACY OF CARDIAC RESYNCHRONISATION THERAPY FOR PATIENTS WITH HEART FAILURE IN THE POPULATION OF LATVIA}

Summary of the Doctoral Thesis for obtaining the degree of a Doctor of Medicine

Specialty - Internal Medicine, Cardiology

Riga, 2019 
The Doctoral Thesis has been elaborated at the Latvian Centre of Cardiology, Pauls Stradiņš University Hospital, Rīga.

Scientific supervisors:

Dr. med., Professor Oskars Kalējs,

Department of Internal Diseases of Rīga Stradiņš University, Latvia

Dr. med., Member of the Latvian Academy of Sciences,

Professor Aivars Lejnieks,

Department of Internal Diseases of Rīga Stradiņš University, Latvia

Scientific advisors:

Medical statistics consultants:

Dr. sc. soc., Andrejs Ivanovs,

Rīga Stradiņš University, Head of the Statistics Unit, Latvia

Kristīne Spalva,

Rīga Stradiņš University, Latvia

Official reviewers:

Dr. med., Assistant Professor Andris Skride,

Department of Internal Diseases of Rīga Stradiņš University, Latvia

Dr. med., Vilnis Dzērve-Tāluts,

University of Latvia, Institute of Cardiology and Regenerative medicine,

Latvia

Dr. med., Professor Aras Puodziukynas,

Head of the Unit of Cardiac Arrythmias, Department of Cardiology

Hospital of Lithuanian University of Health Sciences "Kauno klinikos",

Lithuania

Defence of the Doctoral Thesis will take place at the public session of the Doctoral Council of Medicine on 27 January 2020 at 15.00 in

Hippocrates Lecture Theatre, 16 Dzirciema Street, Rīga Stradiņš University.

The Doctoral Thesis is available in the RSU library and at RSU webpage: www.rsu.lv.

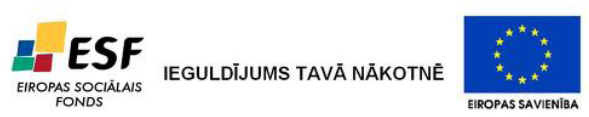

The doctoral thesis was carried out under financial support of the European Social Fund project "Support for doctoral study programmes and the acquisition of a scientific degree at Rīga Stradiņš University"

Secretary of the Doctoral Council:

Dr. med., Associate Professor Inga Stukiēna 


\section{CONTENTS}

Abbreviations used in the paper................................................................

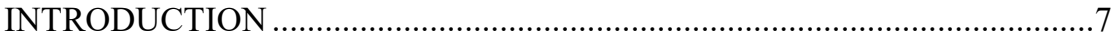

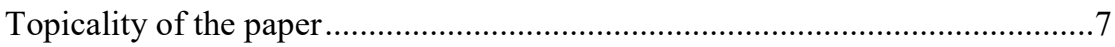

Novelty and practical importance of the paper .................................................10

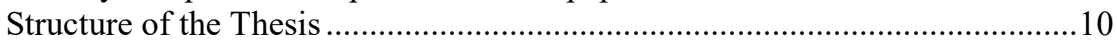

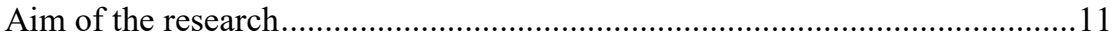

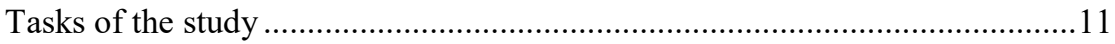

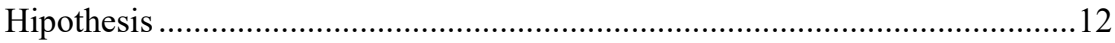

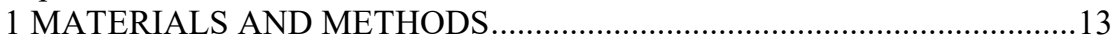

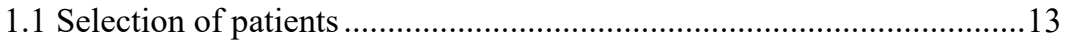

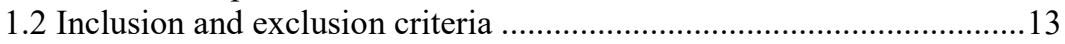

1.3 Statistical analysis of the research data ..................................................14

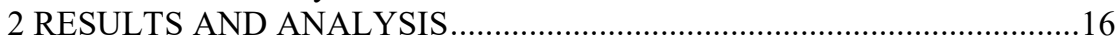

2.1 General characteristics of the study population.....................................16

2.2 Efficacy and safety of cardiac resinchronisation therapy......................18

2.3 Effect of clinical factors on cardiac resinchronisation therapy .............23

2.4 Effect of ECG on cardiac resinchronisation therapy .............................29

2.5 Effect of EchoCG on cardiac resinchronisation therapy .........................31

2.6 Effect of clinical factors on cardiac resinchronisation therapy ...............35

2.7 Effect of cardiac resinchronisation devices on cardiac

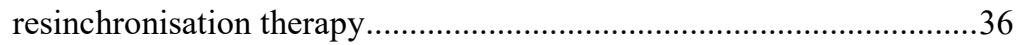

2.8. Prognostic parameters of effective cardiac resinchronisation

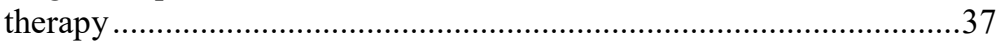

3 DISCUSSION

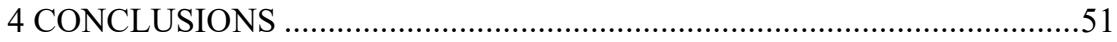

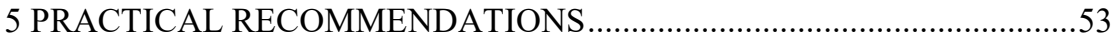

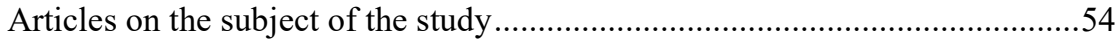

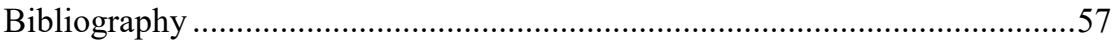




\section{Abbreviations used in the paper}

ACC

ACEI

AHA

ARB

AV

$\mathrm{BAB}$

BiVV

BMI

BNP

$\mathrm{CaCB}$

CHD

$\mathrm{CHF}$

$\mathrm{cm}$

CMP

CRO

CRT

CRT-D

CRT-P

CS

CV

EchoCG

ECG

EDD
American College of Cardiology

angiotensin-converting enzyme inhibitor/s

American Heart Association

angiotensin receptor blocker/s

atrioventricular

beta-adrenergic blocking agent/s

biventricular

body mass index $\left(\mathrm{kg} / \mathrm{m}^{2}\right)$

brain natriuretic peptide

calcium channel blockers

coronary heart disease

chronic heart failure

centimetres

cardiomyopathy

C-reactive protein

cardiac resynchronisation therapy/device

implantable device, which combines functions of

resynchronisation therapy and an implantable

cardioverter defibrillator

implantable device, which combines functions of resynchronisation therapy and a cardiac pacemaker coronary sinus

cardiovascular

echocardiogram

electrocardiogram

left ventricular end diastolic diameter 


$\begin{array}{ll}\text { EF } & \text { ejection fraction } \\ \text { ESD } & \text { left ventricular end systolic diameter } \\ \text { FC } & \text { funcional class } \\ \text { GFR } & \text { glomerular filtration rate } \\ \text { g/dl } & \text { grams per decalitre } \\ \text { Hb } & \text { haemoglobin } \\ \text { HF } & \text { heart failure } \\ \text { ICD } & \text { an implantable cardioverter defibrillator } \\ \text { IVMD } & \text { interventricular mechanic dyssynchrony } \\ \text { IQR } & \text { interquartile range } \\ \text { kg/m }{ }^{2} & \text { kilograms per cubic meter } \\ \text { LAO } & \text { left anterior oblique } \\ \text { LAVI } & \text { left atrial volume index } \\ \text { LBBB } & \text { left His bundle branch block } \\ \text { LVH } & \text { left ventricular hypertrophy } \\ \text { LV } \rightarrow \text { RV } & \text { left ventricle before right ventricle } \\ \text { ml } & \text { millilitres } \\ \text { mm } & \text { millimetres } \\ \text { mm Hg } & \text { millimetres of mercury } \\ \text { Mon } & \text { month } \\ \text { MR } & \text { mitral regurgitation } \\ \text { MRA } & \text { mineralocorticoid receptor antagonist/s } \\ \text { ms } & \text { milliseconds } \\ \text { NASPE } & \text { North American Society of Pacing and } \\ \text { NT-proBNP } & \text { Electrophysiology } \\ \text { OPHA } & \\ & \text { N-terminal pro-brain natriuretic peptide } \\ & \end{array}$


QRS

$\mathrm{p}$

$\mathrm{pg} / \mathrm{ml}$

RAO

RBBB

$\mathrm{RV} \rightarrow \mathrm{LV}$

SBP

SD

SE

TC

VAD

vs.

VV

$\mathrm{x} / \mathrm{min}$

X-ray

P, P-R, Q, q, QRS, RR

QRS complex in an electrocardiogram

$5 \%$ statistical error probability

picograms per millilitre

right anterior oblique

right His bundle branch block

right ventricle before left ventricle

systolic blood pressure

standard deviation

standard error

total cholesterol

ventricular assist devices

against (versus)

interventricular

beats per minute

X-ray image/X-ray examination

markings of teeth and intervals in

an electrocardiographic image 


\section{INTRODUCTION}

\section{Topicality of the paper}

Heart failure (HF) is a pathophysiological condition when disturbance of cardiac functions lead to failure of the heart to pump and to eject blood in accordance with requirements of metabolising tissues (McMurray et al., 2012). The disease is associated with reduced ability of heart to ensure an adequate blood supply throughout the body; thus, leading to the progression of exhaustion, weakness, increase of shortness of breath, formation of congestive symptoms in lungs in a case of minor or low strain and/or in a state of rest, development of hepatomegaly, and swelling of legs. The listed symptoms affect quality of life. Such patients have a significantly increased risk of life-threatening arrhythmias, and a cause of death usually is progression of heart failure and/or ventricular arrhythmias (ventricular tachycardia and ventricular fibrillation) (McMurry et. al., 2012).

In some patients, development of heart failure is caused by electric dyssynchrony between right and left ventricular contractions (uncoordinated contraction of both ventricles). In ECGs it appears in a form of intraventricular conduction disorders through His's bundle main branches, in case of forming of full left His bundle branch block (LBBB) or full right His bundle branch block (RBBB). Electrical and mechanic dyssynchronies result in cardiac contractile dysfunction, ventricular cavity dilation, and decline of cardiac contractile function (Mann, 2011; McMurray et al., 2012).

In patients with moderate to severe HF, pharmacological therapy is often ineffective because it fails to prevent the resulting uncoordinated ventricular contraction. For over a decade drug therapy in the world is accompanied by cardiac resynchronisation devices. Cardiac resynchronisation therapy (CRT) helps restore proper coordination between atria and ventricles of the heart, 
ensures agreed contractions of both ventricles, preventing a patient from ventricular electrical dyssynchrony, which is one of the indicators of unfavourable prognosis of HF (Dickstein et.al., 2008).

Selection of patients for implantation of a CRT device is based on three selection criteria - HF class in line with NYHA (New York Heart Association), width of QRS complex (QRS $\geq 120 \mathrm{~ms}$ according to electrocardiographic data), and Echo cardiographic (EchoCG) parameters - left ventricular ejection fraction $(\mathrm{EF}) \leq 35 \%$ and left ventricular end diastolic diameter (EDD) $\geq 55 \mathrm{~mm}$ measurements. Regardless of the above, approximately in $25-30 \%$ of patients cardiac resynchronisation devices do not ensure the desired effect in reduction of HF and delay of the progression (McMurray et al., 2012; Dickstein et al., 2008).

In the world, CRT method has been used in treatment of patients with heart failure since 2002 (ACC/AHA /NASPE Guidelines, 2002). In Latvia, the first CRT device was implanted in 2006 (J. Ansabergs N. Nesterovičs, M. Blumbergs). In recent years, the number of patients with implanted CRT devices has grown in Latvia (until 2012 CRT implantations were performed only in P. Stradins Clinical University Hospital), but it is still insufficient. In 2011 in Latvia, the total of 55 CRT devices were implanted, of which only half of the patients still have a preserved sinus rhythm, in 2012 - 73 CRT devices and in 2013 - 63 CRT devices were implanted (see Figure 1). When comparing data of Latvia with the data of other European countries, we no not certainly rank among the leaders. On average, 50-150 CRTs per 1 million population per year are being implanted in patients in Eastern Europe, while in Western Europe the number reaches 150-220 of CRT devices per 1 million of population per year (Arribas et al., 2014). In 2013 in Latvia, 28 CRT devices were implanted per 1 million of population. 
Until now, no prospective or retrospective analysis of efficiency of CRT devices have been carried out assessing CRT-induced improvements in treatment of patients with HF. Lack of the expected CRT efficiency in randomised multicentre trials is associated with improper selection of patients, sub-optimal positioning of left ventricular electrode, inadequate choice of medications, as well as unjustified programming of the CRT device. CRT clinical efficiency and inefficiency criteria have not been evaluated also in Latvia. There are still many patients in Latvia for whom the CRT implantation could reduce clinical manifestations of HF, improve their quality of life, as well as reduce mortality. This would reduce the work load of medical emergency service providers, duration of hospital stays and bed-day costs, as well as high cost-burden of treatment of acute heart failure to society.

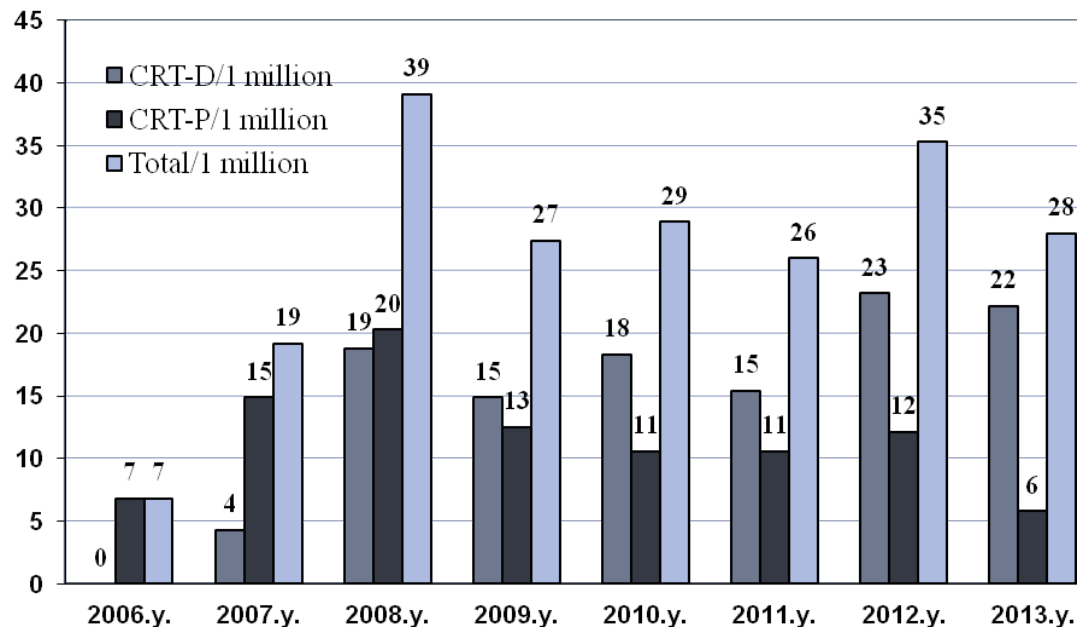

Figure 1 Implantations of cardiac resynchronisation devices in Latvia per 1 million of population in the period from 2006 to 2013 CRT-D - cardiac resynchronisation devices with defibrillation function CRT-P - cardiac resynchronisation devices without defibrillator function 


\section{Novelty and practical importance of the paper}

A study in assessment of efficiency of CRT devices in patients with HF with preserved sinus rhythm assessing the CRT-driven improvement of treatment was carried out for the first time in Latvia.

CRT efficiency-determining parameters for mitigation of ventricular interventricular dyssynchrony, its prevention and therefore positive remodelling of the heart and further stabilisation of clinical course of HF were assessed. Localisation of left ventricular electrode in coronary sinus branches was analysed. A set of CRT device programming parameters was analysed developing recommendations for programming after implantation of a CRT device.

The study allows assessment and specification of indications for application of CRT in patients with HF with interventricular dyssynchrony.

In Latvia the method is used by a number of certified technicians who have actively participated in the study and improved their skills and knowledge during its course. The developed recommendations for selection of patients with HF and improvement of further course of treatment after implantation of CRT will help refine selection of patients and avoid the relatively high number of non-responders in Latvia.

\section{Structure of the Thesis}

The Doctoral Thesis has been written in Latvian, in length of 130 pages. The Paper contains one appendix. The Paper follows a traditional design, including abstracts in Latvian and English, introduction, topicality, novelty and practical importance of the study, aim of the study, tasks, hypothesis, literature review, materials and methods, results, discussion, conclusions, list of articles 
and reports, as well as bibliography consisting of 82 sources. The thesis contains 57 tables and 38 figures.

\section{Aim of the research}

The study aims to analyse progression of treatment and factors affecting its efficacy in HF patients with moderate and severe clinical course using implantable cardiac resynchronisation therapy devices.

\section{Tasks of the study}

1. To evaluate the efficiency of CRT device implantation in patients with moderate and severe HF within a two-year period.

2. To evaluate change in subjective and objective efficiency criteria of HF patients and longitudinal safety of a CRT device.

3. To evaluate differences between groups of CRT responders and nonresponders by comparing data to the initial clinical parameters.

4. To determine differences in visualised EchoCG parameters in high and low CRT efficiency groups.

5. To evaluate differences between groups of CRT responders and nonresponders by analysing EchoCG visualised parameters and laboratory values.

6. To study the impact of CRT implantation technique and programmed parameters on CRT efficiency.

7. To identify dominant factors of longitudinal CRT efficiency, according to study data prior to the implantation of a CRT device. 


\section{Hipothesis}

In application of cardiac resynchronisation devices for patients with moderate and severe heart failure, correlation of diagnostic imaging, enhanced laboratory examinations, localisation of ventricular electrodes, and programming of a cardiac resynchronisation device plays an important role in assessment of selection criteria and efficiency of the complex therapy. 


\section{MATERIALS AND METHODS}

\subsection{Selection of patients}

The study included 50 patients with heart failure who had undergone implantation of cardiac resynchronisation device (CRT) at Pauls Stradins Clinical University Hospital in the period between June 2009 to March 2012.

The study analysed course of treatment for HF patients with moderate and severe clinical course, resistance to pharmacotherapy, exploring predisposing factors for effective cardiac resynchronisation therapy.

This clinical, longitudinal, prospective, two-arm study has received approval from the Ethics Committee for clinical and physiological studies, clinical research of medicinal and pharmaceutical products of the Research Institute of Cardiology of the University of Latvia.

\subsection{Inclusion and exclusion criteria}

CRT implantation was performed in patients on the basis of heart failure treatment guidelines and amendments thereto published by the European Society of Cardiology in 2008 and 2010. Namely, in patients with severe heart failure (with NYHA Class III and outpatiently with NYHA Class IV), with optimal medication therapy (OMT), QRS $\geq 120 \mathrm{~ms}$ and $\mathrm{EF} \leq 35 \%$ and moderate heart failure (with NYHA Class II), if patients receiving OMT, had sinus rhythm, $\mathrm{EF} \leq 35 \%$ and $\mathrm{QRS} \geq 150 \mathrm{~ms}$ (Dickstein et al., 2008; Dickstein et al., 2010).

All patients were informed on tactics and possible complications of CRT implantation, as well as on further programming of a CRT device, development of a plan for assessment of health status and subsequent treatment recommendations. In case of agreement, patients signed a special CRT 
implantation consent form, which is approved for the use in Pauls Stradins Clinical University Hospital.

All patients enrolled in the study had preserved sinus rhythm, and all of them underwent implantation of a CRT device with three electrodes. Thus, all patients were provided with both atrial and ventricular pacing. All patients received OMT for treatment of heart failure.

The study did not include CRT patients with permanent atrial fibrillation to whom sinus rhythm restoration and maintenance was impossible or did not give stable results. Further analysis of the study excluded those patients who underwent atrioventricular connection radio frequency catheter ablation, heart transplantation or a surgery of a ventricular assist device (VAD).

\subsection{Statistical analysis of the research data}

The statistical analysis of the data was performed using the SPSS programme (IBM SPSS Statistics Version 23, SPSS inc., USA). The test results $\mathrm{p}<0.05$ were found to be statistically significant. Quantitative variables were defined through the arithmetic mean and standard deviation (SD). In cases of significant standard error (SE), if the data did not meet normal distribution interquartile range (IQR) and median were assessed. Assessment of qualitative differences of patient groups was determined in \%. Proportional data compliance with normal distribution was determined using Mann-Whitney U-test, and differences between the two varied quantitative data using Wilcoxon signed ranks test. Comparison of normal deviation quantitative variables was performed using Independent samples t-test and differences between the two quantitative variables was performed using Paired samples t-test. Link between quantitative variables was determined by the Spearman and Pearson rank-order correlation coefficient tests, in compliance with the test application procedures. Correlation 
between the two variables was analysed using the logistic regression method. Cathegorical variables were compared using Pearson's chi-square $\left(\chi^{2}\right)$ indicator or Fisher's exact test in compliance with the test application procedures. 


\section{RESULTS AND ANALYSIS}

\subsection{General characteristics of the study population}

The research includes 50 patients who meet the inclusion and exclusion criteria, of which 48 comply with the protocol of the study, the data of which have been used for further study of the research results. Two patients were excluded form the further study due to one having had atrioventricular connection radio frequancy catheter ablation due to frequent atrial fibrilation paroxisms and the other patient had undergone a surgery of VAD device.

Demographics, risk factors, cardiovascular anamnesis, hemodinamics, objective parameters and prehospitalisation therapy of the entire study cohort $(\mathrm{n}=48)$ have been represented in Table 2.1. Prior to implantation of a CRT device, ACEI/ARB was not used by $5(10.4 \%)$ patients due to low arterial blood pressure, BAB was not used by $2(4.2 \%)$ patients due to low heart rate, aldosterone receptor antagonists were not used by 7 (14.6\%) patients due to low arterial blood pressure and GFA.

Table 2.1

Phenotypic characterisation of patient population

\begin{tabular}{ll}
\hline Parameters & \\
\hline Age (years), mean \pm SD & $64.85 \pm 9.49$ \\
Gender - male, $\mathrm{n}(\%)$ & $33(68.8)$ \\
BMI $\left(\mathrm{kg} / \mathrm{m}^{2}\right)$, mean $\pm \mathrm{SD}$ & $29.52 \pm 4.92$ \\
Adiposity $\left(\mathrm{BMI} \geq 30 \mathrm{~kg} \mathrm{/} \mathrm{m}{ }^{2}\right), \mathrm{n}(\%)$ & $21(43.8)$ \\
History of smoking, $\mathrm{n}(\%)$ & $34(70.8)$ \\
Diabetes, $\mathrm{n}(\%)$ & $9(18.8)$ \\
Arterial hypertension, $\mathrm{n}(\%)$ & $18(37.5)$ \\
$\mathrm{SBP}(\mathrm{mm} \mathrm{Hg})$, mean $\pm \mathrm{DS}$ & $125.96 \pm 15.77$ \\
$\mathrm{DBP}(\mathrm{mm} \mathrm{Hg})$, mean $\pm \mathrm{DS}$ & $75.52 \pm 8.33$ \\
$\mathrm{SBP}>140(\mathrm{~mm} \mathrm{Hg}), \mathrm{n}(\%)$ & $15(31.3)$ \\
\hline
\end{tabular}




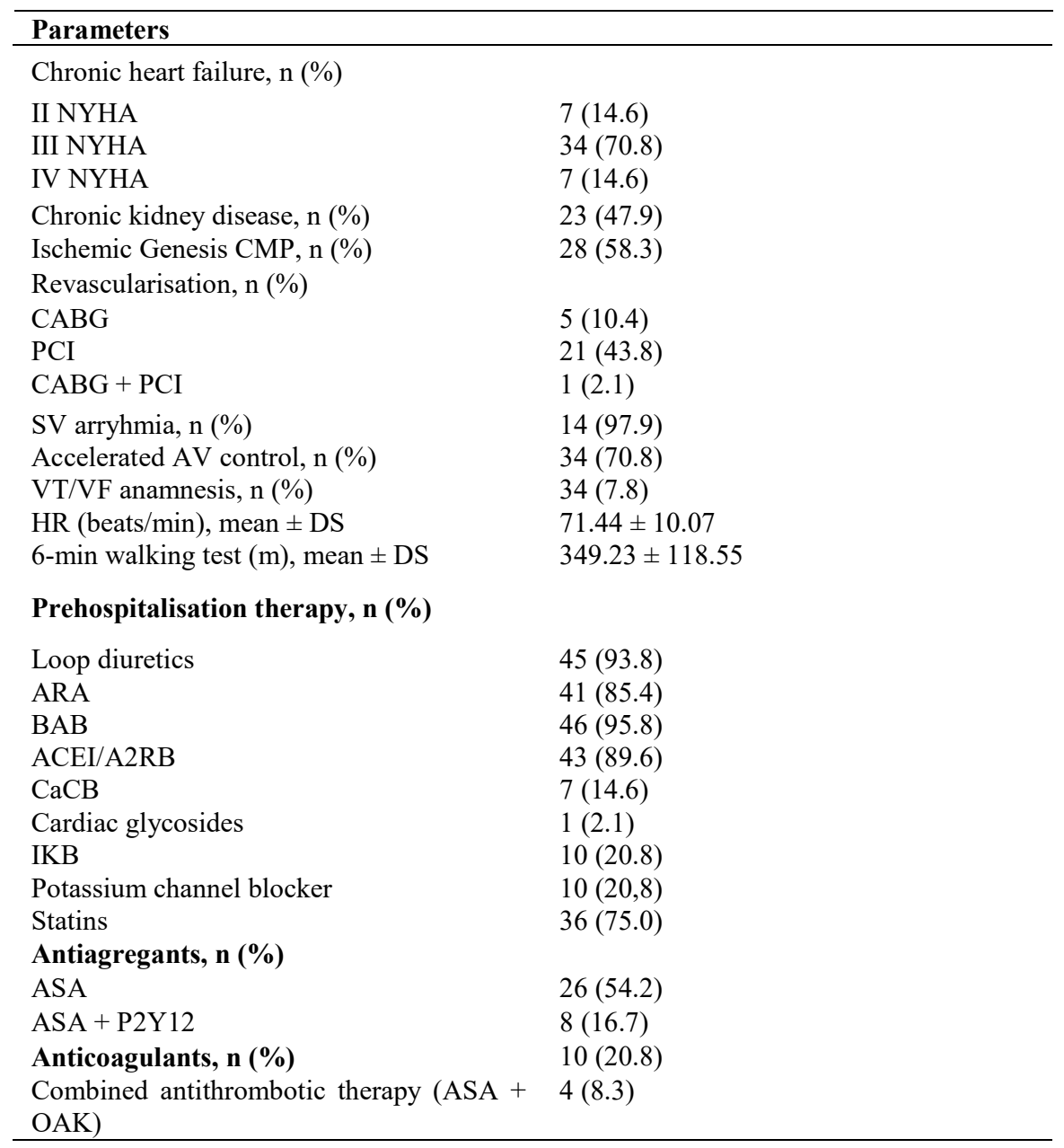

SD - standard deviation, BMI - body mass index, SBP - systolic blood pressure,

DBP diastolic blood pressure, NYHA - New York Heart Association, CMP - cardiomyopathy, $\mathrm{PCI}$ - percutaneous coronary intervention, CABG - coronary artery bypass grafting, $\mathrm{SV}$ - supraventricular, $\mathrm{AV}$ - atrioventricular, $\mathrm{VT} / \mathrm{VF}$ - ventricular tachycardia/fibrilation, HR - heart rate, ARA - aldosterone receptor antagonists; $\mathrm{BAB}$ - beta-adrenergic blocking agents; ACEI - angiotensinconverting enzyme inhibitors; ARB - angiotensin receptor blockers; $\mathrm{CACB}$ - calcium channel blockers; IKB - If channel blockers; ASA - acetylsalicylic acid; OAK - oral anticoagulants. 


\subsection{Efficacy and safety of cardiac resinchronisation therapy}

Initial left ventricular ejection fraction (EF) of the study population, according to the EchoCG data was on average $24.81 \pm 5.23 \%$; after CRT implantation. EF showed statistically significant development (Figure 2.1).

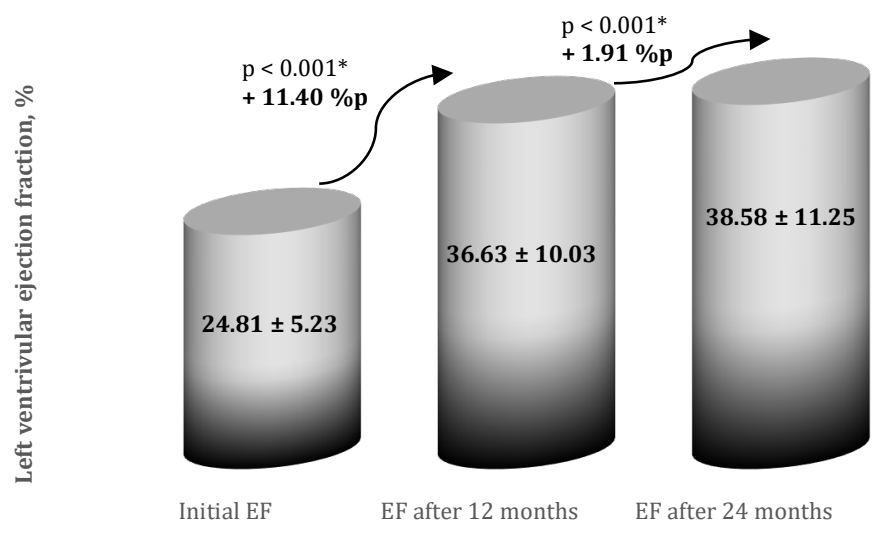

Figure 2.1 Left ventricular ejection fraction before and after CRT implantation

* Paired samples $t$-test was used for data calculations $\mathrm{EF}-$ ejection fraction. $\mathrm{CRT}$ - cardiac resynchronisation therapy

High cardiac resynchronisation therapy efficiency ( $\Delta \mathrm{EF}$ improvement $\geq 10 \%$ ) after 12 months was observed in $23(48.9 \%)$ patients and after 24 months in $27(60.0 \%)$ patients, defined as group A or CRT responders (Figure 2.2). Patients not conforming to the therapeutic effects $(\Delta \mathrm{EF}$ improvement $<10 \%)$ were defined as group B or CRT non-responders. 


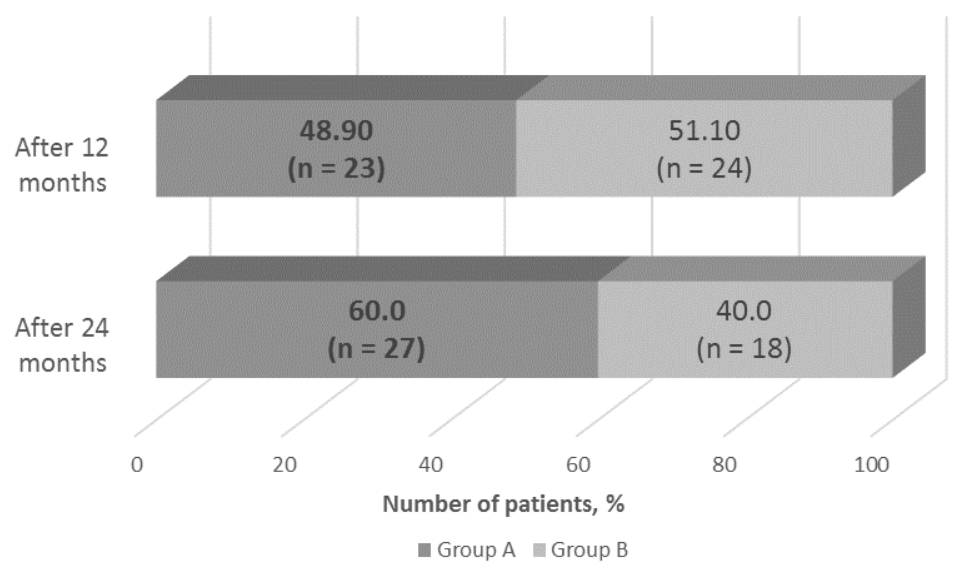

Figure 2.2 Evaluation of cardiac resynchronisation therapy efficiency ( $\triangle \mathbf{E F}$ improvement $\geq 10 \%$ ) during patient testing

$\Delta \mathrm{EF}$ - changes in ejection fraction

Cardiovascular death due to CHF decompensation occurred in three patients: for one patient $(2.1 \%)$ in three months, for two patients $(4.2 \%)$ in 24 months; thus, the number of patient data analysed within the 12-month visitation was 47 , and within 24 months - 45 patients. Device-related complications and bleeding was not observed. Post-CRT device implantation patients were statistically significantly less frequently admitted to hospital (Figure 2.3). 


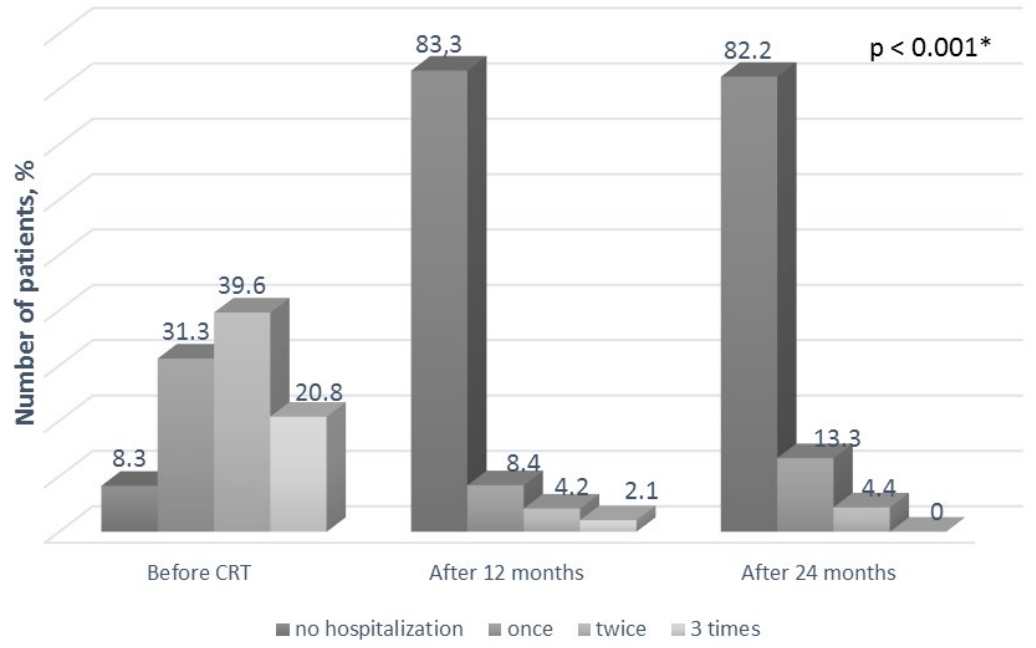

Figure 2.3 Frequency of hospitalisation before and after CRT implantation

* Wilcoxon signed ranks test was used for data calculations CRT - cardiac resynchronisation therapy

After CRT implantation, according to NYHA classification, patients' functional class (FC) improved statistically significantly. Upon hospital admission, FC IV was observed in 7 (14.6\%), in 12 months - 2 (4.3\%), 24 months later in $1(2.2 \%)$ patient; $\mathrm{p}<0.001$ (Figure 2.4). 


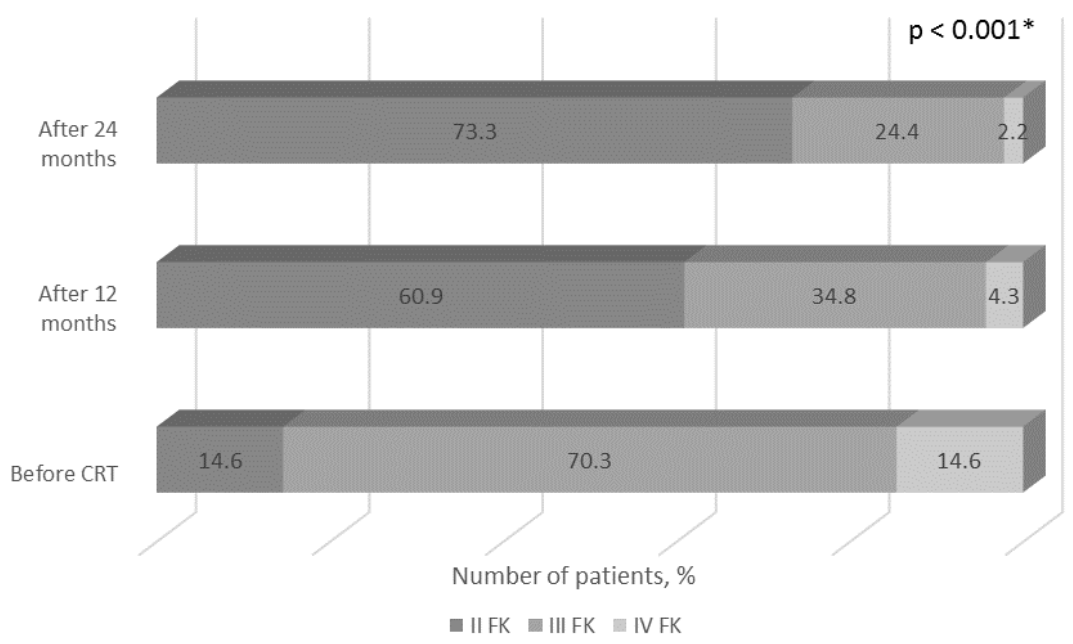

Figure 2.4 NYHA funcional class before and after CRT implantation

* Wilcoxon signed ranks test was used for data calculations

NYHA - New York Heart Association, CRT - cardiac resynchronisation therapy

A year after cardiac resynchronisation therapy device implantation $(\mathrm{n}=47), 6$-minute walking test statistically significantly improved by 113.85 metres; $\mathrm{p}<0.001$; however, after 24 months $(\mathrm{n}=44)$ it improved by 147.25 metres; $\mathrm{p}<0.001$.

After CRT implantation, mean BNP median statistically significantly decreased form $939.00 \mathrm{pg} / \mathrm{ml}[517.50 ; 1769.25]$ to $320.00 \mathrm{pg} / \mathrm{ml}$ [148.00; 653.00] after 12 months $(\mathrm{p}<0.001)$ and $189.00 \mathrm{pg} / \mathrm{ml}$ [100.50; 518.50] after 24 months $(\mathrm{p}<0.001)$ (Figure 2.5). 

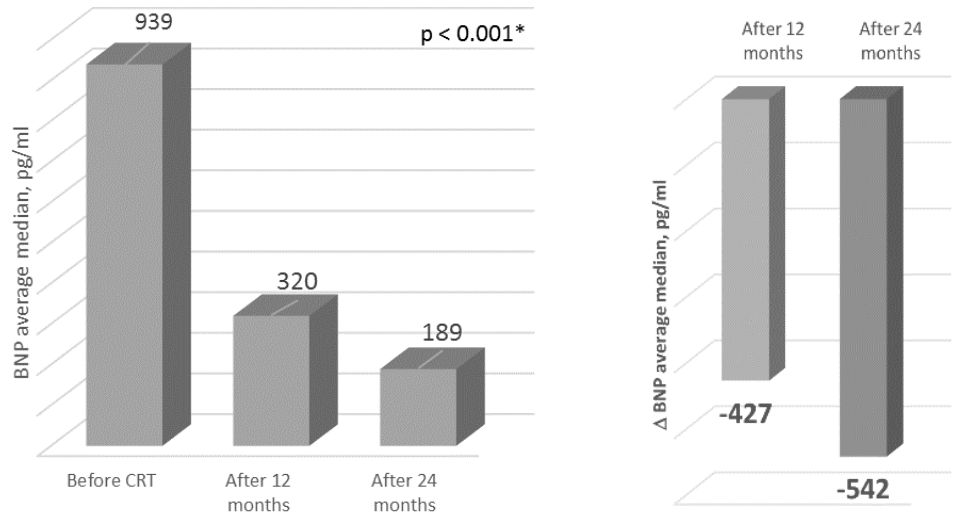

Figure 2.5 BNP and its changes before and after CRT implantation

* Wilcoxon signed ranks test was used for data calculations

$\mathrm{BNP}$ - brain natriuretic peptide, CRT - cardiac resynchronisation therapy, $\Delta$ - changes

Immediately after cardiac resynchronisation therapy device implantation, the mean QRS width significantly decreased by $-15.50 \mathrm{~ms} ; \mathrm{p}<0.001$, after 12 months the mean QRS width significantly decreased by $-18.75 \mathrm{~ms} ; \mathrm{p}<0.001$; however, after 24 months by $-17.31 \mathrm{~ms} ; \mathrm{p}<0.001$ (Paired samples t-test was used for data calculations).

EchoCG parameters decreased statistically significantly 12 months after CTR implantation - left ventricular end diastolic diameter (EDD) by $-4.89 \mathrm{~mm}$ $(\mathrm{p}=0.006)$ and after 24 months by $-8.00 \mathrm{~mm}(\mathrm{p}<0.001)$, corespondingly left ventricular end systolic diameter $(\mathrm{ESD})$ by $-8.47 \mathrm{~mm}(\mathrm{p}<0.001)$ and -10.65 $\mathrm{mm}(\mathrm{p}<0.001)$, left atrial volume index (LAVI) decreased by $-4.07 \mathrm{ml} / \mathrm{m}^{2}$ $(\mathrm{p}=0.138)$ and $-6.39 \mathrm{ml} / \mathrm{m}^{2}(\mathrm{p}=0.009)$ (Figure 2.6). Interventricular mechanic dyssynchrony (IVMD) decreased statistically significantly after 12 months by $-26.33 \mathrm{~ms}(\mathrm{p}<0.001)$ and $-23.84 \mathrm{~ms}(\mathrm{p}<0.001)$ after 24 months of CRT implantation. 


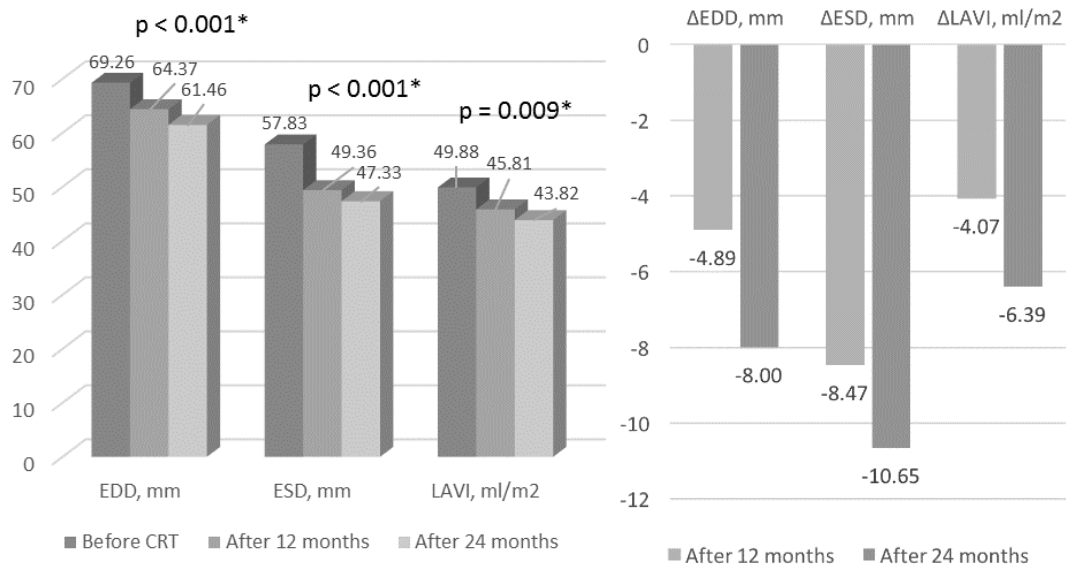

Figure 2.6 EchoCG parameters and their changes before and after CRT implantation

* Paired samples t-test was used in data calculations

EchoCG - echocardiogram, CRT - cardiac resynchronisation therapy,

EDD - left ventricular end diastolic diameter, ESD - left ventricular end systolic diameter, LAVI - left atrial volume index, $\Delta-$ changes

After CRT implantation, degree of wet regurgitation in patients improved statistically significantly; respectively, upon joining the hospital degree III was indicated in 7 (14.6\%), after 12 months in 3 (6.4\%), and after 24 months in $2(4.4 \%)$ patients; $\mathrm{p}<0.001$.

\subsection{Effect of clinical factors on cardiac resinchronisation therapy}

Efficiency of cardiac resinchronisation therapy after two years, based on phenotypic characteristics of the study population has been represented in Table 2.2. The studied phenotypic characteristics had no significant deviation form the one-year cardiac resinchronisation therapy efficiency groups.

Considering individual demographic and anamnesis factors, significantly different trends were observed in CRT responders and non-responders groups. In 12 months, cardiac resinchronisation therapy was statistically significantly 
more prevalent in females $(n=15)$ compared to males $(n=32)$, respectively 11 $(73.3 \%)$ vs. $12(37.5 \%$; $=0.030)$; a similar trend was observed in 24 months as well (Figure 2.7). After 12 months, smokers $(n=34)$ exhibited the trend to less frequently end up in CRT responders group compared to non-smokers $(\mathrm{n}=13)$, respectively $14(41.2 \%)$ vs. $9(69.2 \% ; \mathrm{p}=0.111 ;$ Chi-square test was used for data calculations).

Table 2.2

Anamnesis and demographic data with $\Delta E F \geq 10 \%$ and $\Delta E F<10 \%$ after 24 months

\begin{tabular}{|c|c|c|c|}
\hline Parameter & $\begin{array}{c}\text { Group A } \\
(\mathrm{n}=\mathbf{2 7})\end{array}$ & $\begin{array}{c}\text { Group B } \\
(n=18)\end{array}$ & p value* \\
\hline Age (years), mean $\pm \mathrm{SD}$ & $\begin{array}{c}63.41 \pm \\
9.85\end{array}$ & $65.00 \pm 8.22$ & $0.560 * *$ \\
\hline $\mathrm{BMI}\left(\mathrm{kg} / \mathrm{m}^{2}\right)$, mean $\pm \mathrm{SD}$ & $\begin{array}{c}29.72 \pm \\
5.12\end{array}$ & $29.47 \pm 4.69$ & $0.866^{* *}$ \\
\hline Adiposity $\left(\mathrm{BMI} \geq 30 \mathrm{~kg} / \mathrm{m}^{2}\right), \mathrm{n}(\%)$ & $13(48.1)$ & $7(38.9)$ & $.0,540$ \\
\hline Parameter & $\begin{array}{c}\text { Group A } \\
(\mathbf{n}=\mathbf{2 7})\end{array}$ & $\begin{array}{c}\text { Group B } \\
(\mathrm{n}=18)\end{array}$ & p value* \\
\hline Diabetes, n (\%) & $6(22.2)$ & $1(5.6)$ & 0.215 \\
\hline Arterial hypertension, n (\%) & $18(66.7)$ & $9(50.0)$ & 0.264 \\
\hline \multicolumn{4}{|l|}{ Heart Failure Class, n (\%) } \\
\hline NYHA II & $3(11.1)$ & $4(22.2)$ & 0.466 \\
\hline NYHA III & $21(77.8)$ & $11(61.1)$ & \\
\hline NYHA IV & $3(11.1)$ & $3(16.7)$ & \\
\hline Chronic kidney disease, n (\%) & $11(40.7)$ & $10(55.6)$ & 0.329 \\
\hline \multicolumn{4}{|l|}{ Revascularisation, n (\%) } \\
\hline CABG & $2(7.4)$ & $1(5.9)$ & \\
\hline PCI & $9(33.3)$ & $11(64.7)$ & 0.078 \\
\hline $\mathrm{CABG}+\mathrm{PCI}$ & 0 & $1(5.9)$ & \\
\hline SV arrhythmia, n (\%) & $19(70.4)$ & $12(66.7)$ & 0.793 \\
\hline Accelerated AV control, n (\%) & $9(33.3)$ & $5(27.8)$ & 0.693 \\
\hline VT/VF anamnesis, n (\%) & $23(85.2)$ & $15(83.3)$ & 0.867 \\
\hline
\end{tabular}

* Chi-square test and **Independent samples $t$ - test were used for data calculations.

$\Delta \mathrm{EF}-$ changes in ejection fraction, group $\mathrm{A}-\Delta \mathrm{EF}$ improvement $\geq 10 \%$, group $\mathrm{B}-\triangle \mathrm{EF}$ improvement $<10 \%$, BMI - body mass index, NYHA - New York Heart Association, PCI - percutaneous coronary intervention, CABG - coronary artery bypass grafting, SV - supraventricular, AV - atrioventricular, VT - ventricular tachycardia, VF - ventricular fibrillation, SD - standard deviation. 


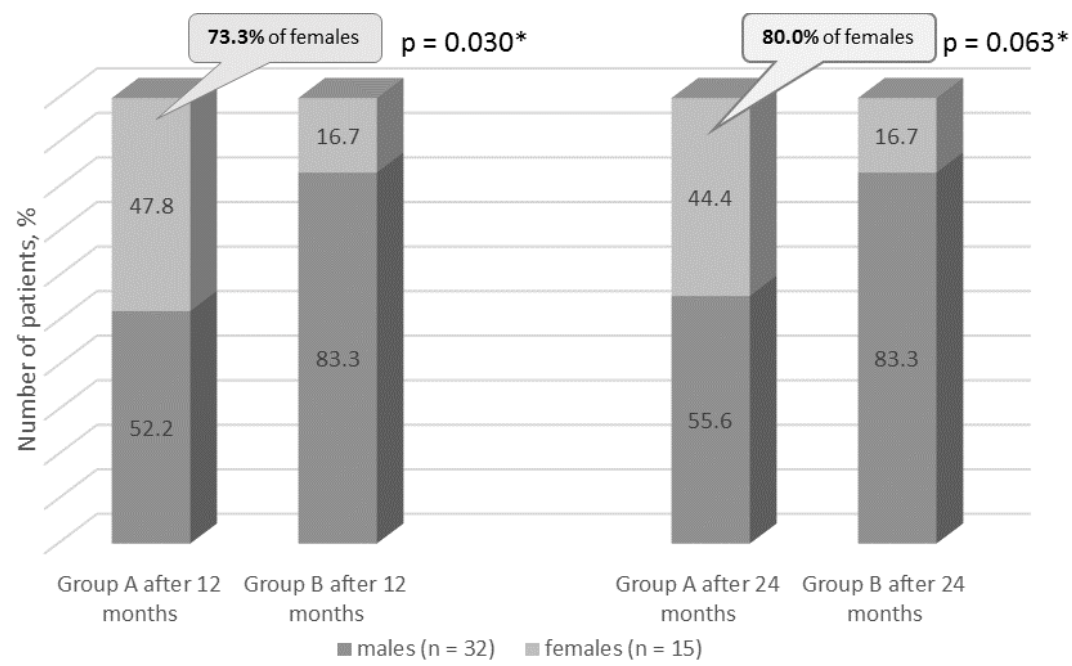

Figure 2.7 Gender impact on efficiency of cardiac resinchronisation therapy after 12 and 24 months

* Chi-square test was used in data calculations

Group $\mathrm{A}-\Delta \mathrm{EF}$ improvement $\geq 10 \%$, group $\mathrm{B}-\Delta \mathrm{EF}$ improvement $<10 \%$

Based on the obtained long-term data (24 months), CRT efficacy was statistically significantly more frequently observed in patients $(n=20)$ with non-ischemic genesis CMP $16(66.7 \%)$ compared to ischemic CMP $(\mathrm{n}=25)$ patients $11(44.0 \% ; \mathrm{p}=0.018)$ (Figure 2.8). Patients $(\mathrm{n}=20)$ without identified revascularisation, after 24 months presented similar data statistically significantly more frequently resulting in CRT responders group compared to patients with prior revascularisation, respectively $16(80.0 \%)$ vs. 11 (42.3\%; $\mathrm{p}=0.030$; Chi-square test was used in data calculations).

Correlation between phenotypic and objective data using cardiac resinchronisation therapy has been represented in Table 2.3. 


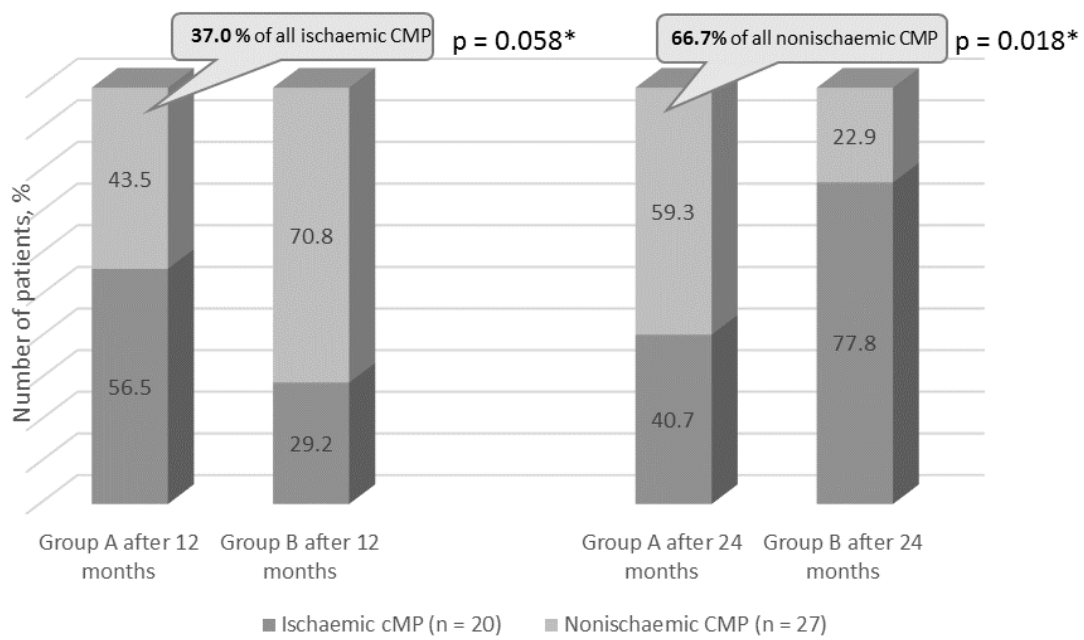

Figure 2.8 Effect of cardiomyopathy genesis on effect of cardiac resinchronisation therapy after 12 and 24 months

* Chi-square test was used in data calculations

Group $\mathrm{A}-\Delta \mathrm{EF}$ improvement $\geq 10 \%$, group $\mathrm{B}-\Delta \mathrm{EF}$ improvement $<10 \%$, $\mathrm{CMP}$ - cardiomyopathy

Table 2.3

Relationship between subjective and objective parameters with $\Delta \mathrm{EF} \geq 10 \%$ and $\Delta E F<10 \%$

\begin{tabular}{|c|c|c|c|c|}
\hline Parameter & $\begin{array}{c}\mathrm{r} \text { factor } \\
\text { after } 12 \\
\text { months }(\mathrm{n}= \\
47)\end{array}$ & p value* & $\begin{array}{c}r \text { factor after } \\
24 \text { months (n } \\
\quad=45)\end{array}$ & p value* \\
\hline $\begin{array}{l}\text { HR (beats/min), mean } \pm \\
\text { DS }\end{array}$ & 0.131 & 0.381 & 0.183 & 0.230 \\
\hline $\mathrm{SBP}(\mathrm{mm} \mathrm{Hg})$, mean \pm DS & 0.307 & 0.036 & 0.436 & 0.003 \\
\hline $\mathrm{DBP}(\mathrm{mm} \mathrm{Hg})$, mean $\pm \mathrm{DS}$ & 0.129 & 0.387 & 0.016 & 0.916 \\
\hline $\begin{array}{l}\text { 6-min walking test }(\mathrm{m}) \\
\text { mean } \pm \mathrm{DS}\end{array}$ & 0.058 & 0.700 & 0.029 & 0.849 \\
\hline CHF, NYHA FC, n (\%) & -0.078 & $0.602 * *$ & 0.053 & $0.731 * *$ \\
\hline $\mathrm{BMI}\left(\mathrm{kg} / \mathrm{m}^{2}\right)$, vid $\pm \mathrm{SD}$ & 0.166 & 0.263 & 0.112 & 0.463 \\
\hline Re-hospitalisation, n (\%) & -0.259 & $0.078 * *$ & 0.294 & $0.050 * *$ \\
\hline
\end{tabular}

* Pearson and **Spearman correlation tests were used in data callculations.

$\triangle \mathrm{EF}$ - ejection fraction change, $\mathrm{HR}$ - heart rate, SBP - systolic blood pressure, DBP - diastolic blood pressure, BMI - body mass index, CHF - chronic heart failure, NYHA - New York Heart Association, FC - functional class. 
After 12 months, statistically significantly higher systolic blood pressure was observed in patients $(\mathrm{n}=23)$ with improved $\Delta \mathrm{EF} \geq 10 \%, 130.70 \pm$ $14.14 \mathrm{~mm} \mathrm{Hg}$ compared to patients $(\mathrm{n}=24)$ with $\Delta \mathrm{EF}<10 \%, 121.06 \pm 16.28$ $\mathrm{mm} \mathrm{Hg} ; \mathrm{p}=0.036$; statistically significant differences remained after 24 months as well, respectively $131.89 \pm 14.73$ vs. $117.78 \pm 14.37 \mathrm{~mm} \mathrm{Hg} ; \mathrm{p}=0.003$ (Figure 2.9)

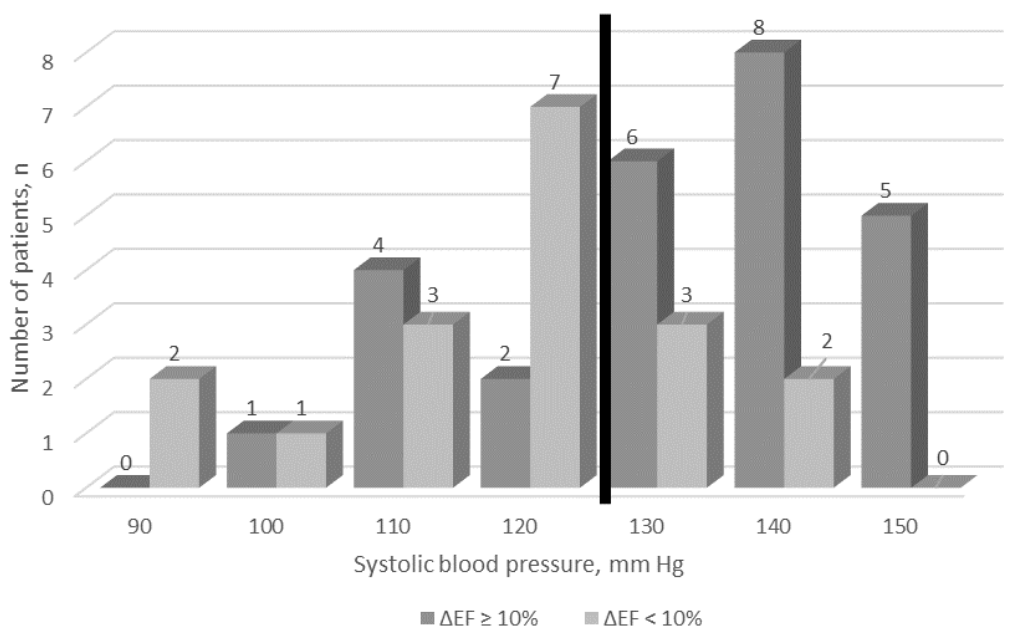

Figure 2.9 Systolic blood pressure based on cardiac resinchronisation therapy efficacy after 24 months

* Independent samples $t$ - test was used for data calculations $\Delta \mathrm{EF}$ - changes in ejection fraction

CRT efficacy after two years dependant on subjective and objective data characterisation of the other patient population has been represented in Table 2.4. The analysed phenotypic data showed little discrepancy with the one-year cardiac resinchronisation therapy efficacy in groups. 
Table 2.4

\section{Difference in objective parameters $\Delta E F \geq 10 \%$ and $\Delta E F<10 \%$ in groups after 24 months}

\begin{tabular}{lccc}
\hline Parameter & $\begin{array}{c}\text { Group A } \\
(\mathbf{n = 2 7 )}\end{array}$ & $\begin{array}{c}\text { Group B } \\
(\mathbf{n}=\mathbf{1 8})\end{array}$ & p value \\
\hline HR (beats/min), mean \pm SD & $72.81 \pm 11.27$ & $69.06 \pm 8.13$ & $0.201^{*}$ \\
SBP (mm Hg), mean \pm SD & $131.89 \pm 14.73$ & $117.78 \pm 14.37$ & $\mathbf{0 . 0 0 3 *}$ \\
DBP (mm Hg), mean \pm SD & $75.56 \pm 8.47$ & $75.28 \pm 8.82$ & $0.917^{*}$ \\
HR $>$ 70 beats/min, n (\%) & $15(55.6)$ & $9(50.0)$ & $0.714^{* *}$ \\
6-min walking test (m), mean & & & \\
\pm SD & $354,04 \pm 112,10$ & $346 \pm 137,91$ & $0,856^{*}$ \\
Hospitalisation prior to CRT & & & \\
implantation, n (\%) & $24(88.9)$ & $17(94.4)$ & $0.640^{* *}$ \\
\hline
\end{tabular}

* Independent samplest-test, ${ }^{* * C h i-s q u a r e ~ t e s t ~ w e r e ~ u s e d ~ i n ~ d a t a ~ c a l c u l a t i o n s . ~}$ $\Delta \mathrm{EF}-$ changes in ejection fraction, group $\mathrm{A}-\Delta \mathrm{EF}$ improvement $\geq 10 \%$, group $\mathrm{B}-\triangle \mathrm{EF}$ improvement $<10 \%$, HR - heart rate, SBP - systolic blood pressure, DBP - diastolic blood pressure, CRT - cardiac resinchronisation therapy, SD - standard deviation.

Patients $(n=23)$ with a trend for a lower systolic blood pressure ( $\mathrm{SBP}<130 \mathrm{~mm} \mathrm{Hg}$ ) upon hospitalisation resulted in CRT responders group much less frequently compared to patients $(\mathrm{n}=24)$ with $\mathrm{SBP} \geq 130 \mathrm{~mm} \mathrm{Hg}$, respectively $8(34.8 \%)$ vs. $15(62.5 \%$; $=0.057)$ after 12 months and 8 $(31.1 \%)$ vs. $19(79.2 \% ; p=0.005)$ after 24 months (Figure 2.10).

Patients less frequently admitted to hospital due to HF decompensation prior to CRT implantation statistically more frequently resulted in CRT responders group after 12 months, respectively $3(75.0 \%)$ - without prior hospitalisation $(n=4)$ vs. $8(53.3 \%)$ - once hospitalised $(n=15)$ vs. 10 $(55.6 \%)$ - twice hospitalised $(\mathrm{n}=18)$ vs. $2(20.0 \%)-$ thrice hospitalised $(\mathrm{n}=10)$; however, statistical significance was not reached $\mathrm{p}=0.191$. A similar trend was observed also after 24 months, respectively $3(75.0 \%)$ - without prior hospitalisation $(n=4)$ vs. $10(71.4 \%)$ - once hospitalised $(n=14)$ vs. $11(64.7 \%)$ - twice hospitalised $(n=17)$ vs. $3(30 \%)$ - thrice hospitalised $(n=10) ; p=0.169$. 
CRT efficacy dependant on prehospitalised medicine did not reach statistical reliability.

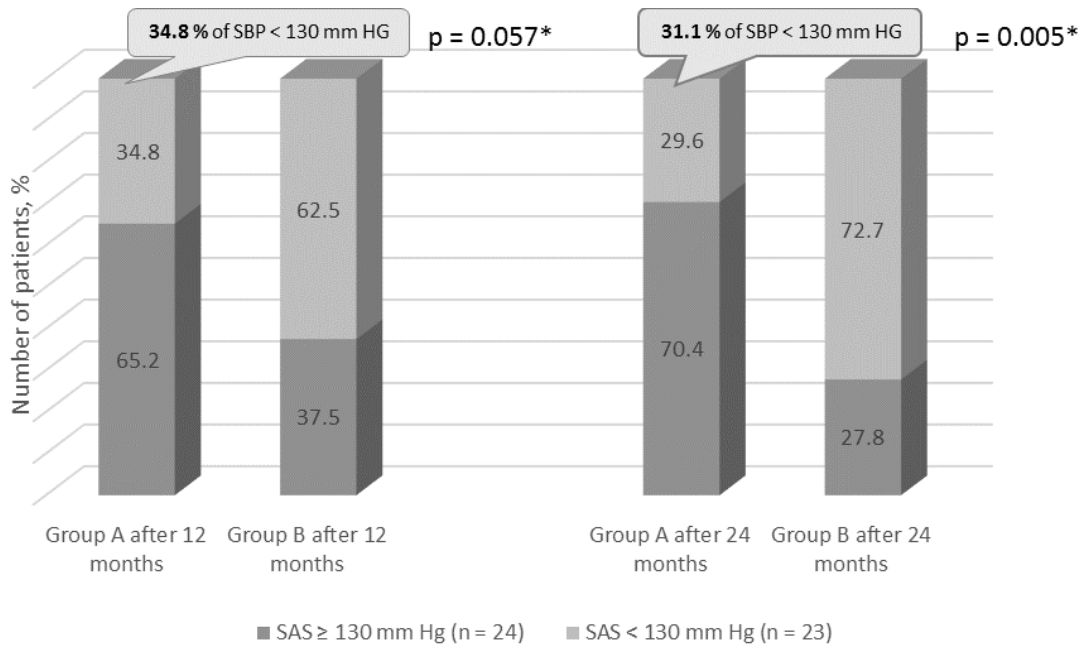

Figure 2.10 Impact of systolic blood pressure on cardiac resinchronisation therapy after 12 and 24 months

* Chi-square test was used in data collections. Group $\mathrm{A}-\Delta \mathrm{EF}$ improvement $\geq 10 \%$, group $\mathrm{B}-\Delta \mathrm{EF}$ improvement $<10 \%$, SBP - systolic blood pressure

\subsection{Effect of ECG on cardiac resinchronisation therapy}

Analysing the impact of the initial mean QRS width on CRT efficiency, a statistically significant moderate positive correlation was observed after 12 months $(\mathrm{r}=0.322 ; \mathrm{p}=0.027)$ and after 24 months $(\mathrm{r}=0.297 ; \mathrm{p}=0.048)$, which indicates on a better response to CRT treatment in patients with a wider QRS (Pearson correlation test was used in data collections).

In patients $(\mathrm{n}=23)$ with improved $\Delta \mathrm{EF} \geq 10 \%$ after 12 months a statistically significant wider mean QRS $177.04 \pm 21.20 \mathrm{~ms}$ was observed compared to patients $(\mathrm{n}=24)$ with $\Delta \mathrm{EF}<10 \%, 162.54 \pm 22.38 \mathrm{~ms} ; \mathrm{p}=0.027$; 
statistically significant differences were observed also after 24 months, respectively $175.15 \pm 20.29$ vs. $161.22 \pm 25.40 \mathrm{~ms} ; \mathrm{p}=0.048$ (Figure 2.11).

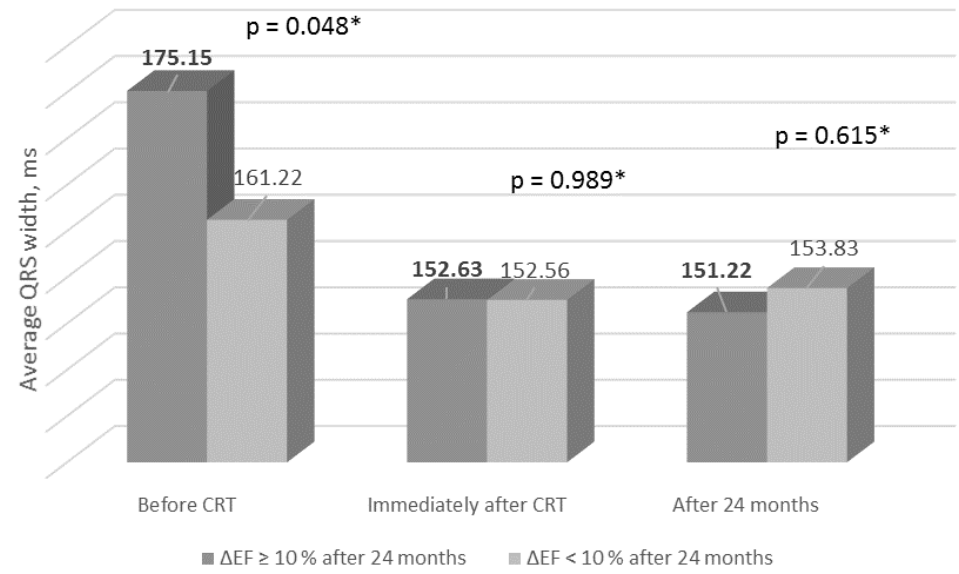

Figure 2.11 QRS width before and after CRT implantation

* Independent samples $\mathrm{t}$-testwas used in data collections

$\Delta \mathrm{EF}$ - changes in ejection fraction, $\mathrm{CRT}$ - cardiac resinchronisation therapy

After 12 months, patients with LBBB after ECG $(\mathrm{n}=36)$ showed improved cardiac resinchronisation therapy statistically significantly more frequently compared to patients with RBBB with His bundle of incomplete LBB $(n=9)$ or dissinchrony of echocardiography without wide QRS $(n=2)$, respectively $22(61.1 \%)$ vs. $1(11.1 \%)$ vs. $0(0 \%)$; $=0.030)$; after 24 months a similar trend was observed, respectively $26(74.3 \%)$ vs. $1(12.5 \%)$ vs. 0 $(0 \% ; \mathrm{p}=0.001)($ Figure 2.12). 


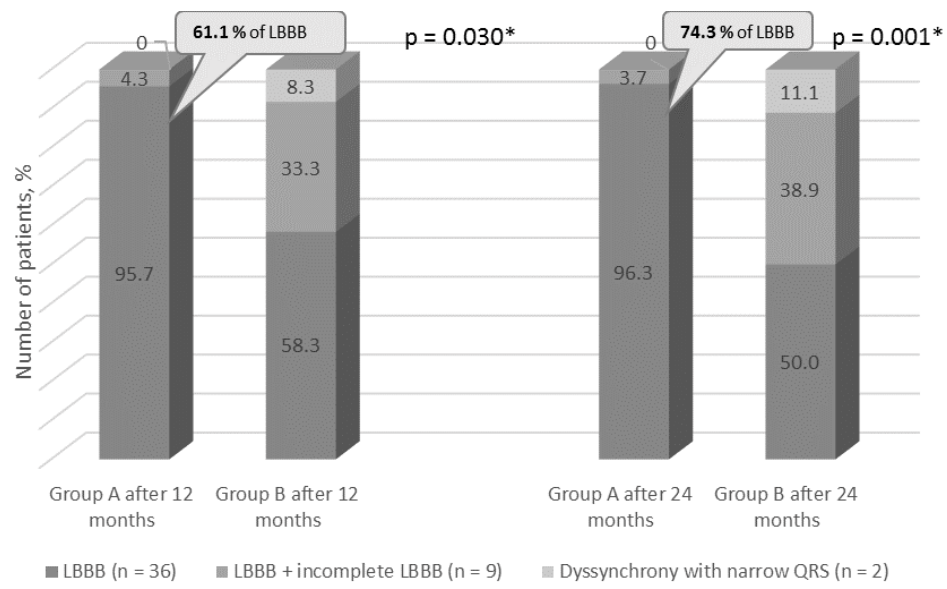

Figure 2.12 Impact of QRS morphology on cardiac resinchronisation therapy after 12 and 24 months

* Chi-square testwas used in data calculations

Group $\mathrm{A}-\triangle \mathrm{EF}$ improvement $\geq 10 \%$, group $\mathrm{B}-\Delta \mathrm{EF}$ improvement $<10 \%$, LBBB - left His bundle branch block, RBBB - right His bundle branch block, EchoCG - echocardiogram

\subsection{Effect of EchoCG on cardiac resinchronisation therapy}

Correlation of echocardiogram parameters and cardiac resinchronisation therapy has been represented in Table 2.5. Analysing the initial IVMD impact on CRT, a statistically significant moderate positive correlation was observed after 12 months $(\mathrm{r}=0.427 \mathrm{p}=0.003)$ and a strong correlation after 24 months $(r=0.560 \mathrm{p}<0.001)$. Patients $(\mathrm{n}=24)$ with a scar in the left ventricle wall resulted in the group of cardiac resinchronisation therapy statistically significantly less frequently $6(26.1 \%)$ compared to patients $(\mathrm{n}=24)$ without a scar $17(73.9 \% ; \mathrm{p}=0.002)$ after 12 months and $7(25.9 \%)$ vs. $20(74.1 \%$; $\mathrm{p}=0.001)$ after 24 months (Chi-square test was used in data calculations). Efficiency of cardiac resinchronisation therapy dependant on myocardial contractility has been respresented in Figure 2.13. 
Table 2.5

Correlation of echocardiogram parameters with $\Delta E F \geq 10 \%$ and $\Delta E F<10 \%$

\begin{tabular}{lcccc}
\hline \multicolumn{1}{c}{ Parameter } & $\begin{array}{c}\text { r coeficient } \\
\text { after 12 } \\
\text { months } \\
(\mathbf{n = 4 7 )}\end{array}$ & p value & $\begin{array}{c}\text { r coefficient } \\
\text { after 24 } \\
\text { months } \\
(\mathbf{n}=\mathbf{4 5})\end{array}$ & p value \\
\hline EDD $(\mathrm{mm})$, mean \pm DS & 0.074 & 0.620 & 0.113 & 0.462 \\
ESD (mm), mean \pm DS & 0.174 & 0.243 & 0.241 & 0.111 \\
LAVI (ml $\left./ \mathrm{m}^{2}\right)$, mean \pm DS & 0.142 & 0.341 & 0.124 & 0.140 \\
MR degree, n $(\%)$ & 0.088 & $0.556^{* *}$ & -0.018 & $0.908^{* *}$ \\
IVMD $(\mathrm{ms})$, mean \pm DS & 0.427 & $\mathbf{0 . 0 0 3}$ & 0.560 & $<\mathbf{0 . 0 0 1}$ \\
\hline
\end{tabular}

* Pearson and **Spearman correlation tests were used in data calculations. $\triangle \mathrm{EF}$ - changes in ejection fraction, $\mathrm{CRT}$ - cardiac resinchronisation therapy, IVMD - interventricular mechānical dyssynchrony, EDD - left ventricular end diastolic diameter, ESD - left ventricular end systolic diameter, LAVI - left atrial volume index, $\mathrm{MR}$ - mitral regurgitation.

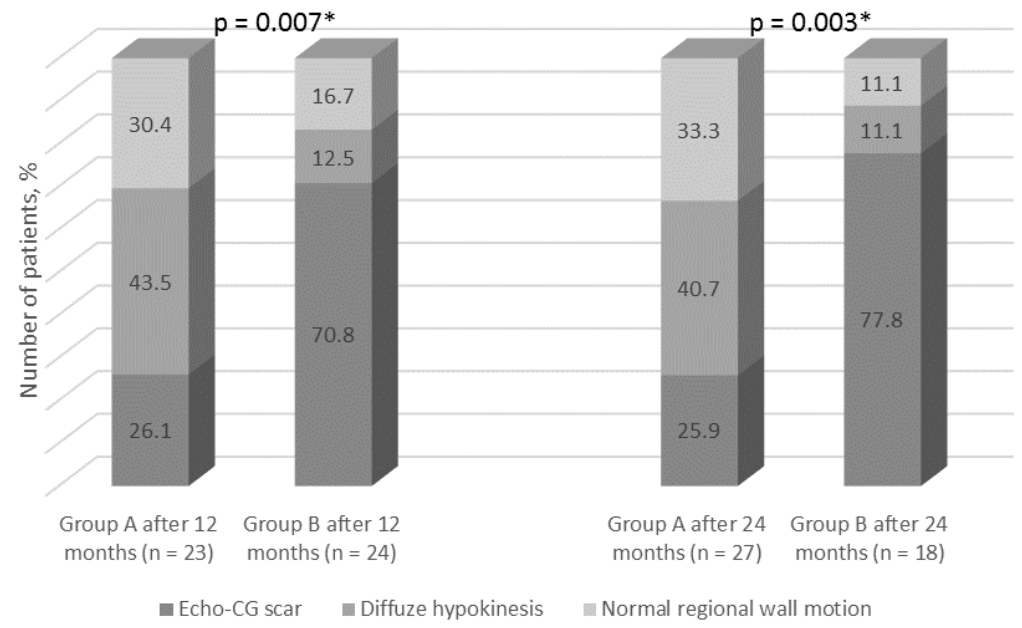

Figure 2.13 Impact of myocardial contractility on cardiac resinchronisation therapy after 12 and 24 months

* Chi-square test wasw used in data calculations

Group $\mathrm{A}-\Delta \mathrm{EF}$ improvement $\geq 10 \%$, group $\mathrm{B}-\Delta \mathrm{EF}$ improvement $<10 \%$, EchoCG - echocardiogram 
Efficiency of cardiac resinchronisation therapy dependant on echocardiographic characteristics of the patient population within a year and two has been represented in Tables 2.6 and 2.7.

Table 2.6

Echocardiogram data with $\Delta E F \geq 10 \%$ and $\Delta E F<10 \%$ after 12 months

\begin{tabular}{|c|c|c|c|}
\hline Parameter & $\begin{array}{c}\text { Group A } \\
(n=23)\end{array}$ & $\begin{array}{c}\text { Group B } \\
(n=24)\end{array}$ & p value \\
\hline MI scars, n (\%) & $6(26.1)$ & $17(70.8)$ & $0.002 *$ \\
\hline MI scar localisation, n (\%) & & & \\
\hline Anterior wall & $3(50.0)$ & $4(23.5)$ & \multirow{4}{*}{$0.019 *$} \\
\hline Top & $1(16.7)$ & $4(23.5)$ & \\
\hline Wide anterior wall & $0(0)$ & $9(52.9)$ & \\
\hline Prosterior wall & $2(33.3)$ & $0(0)$ & \\
\hline $\mathrm{EDD}(\mathrm{mm})$, mean $\pm \mathrm{DS}$ & $69.70 \pm 5.70$ & $68.83 \pm 6.13$ & $0.620 * *$ \\
\hline $\begin{array}{l}\text { EDD after } 12 \text { mon. }(\mathrm{mm}), \\
\text { mean } \pm \text { DS }\end{array}$ & $60.31 \pm 14.22$ & $68.25 \pm 7.34$ & $0.020 * *$ \\
\hline $\mathrm{ESD}(\mathrm{mm})$, mean $\pm \mathrm{DS}$ & $59.13 \pm 6.82$ & $56.58 \pm 7.87$ & $0.243 * *$ \\
\hline $\begin{array}{l}\text { ESD after } 12 \text { mon. (mm), } \\
\text { mean } \pm \text { DS }\end{array}$ & $42.17 \pm 10.91$ & $56.25 \pm 10.24$ & $<0.001 * *$ \\
\hline $\mathrm{LAVI}\left(\mathrm{ml} / \mathrm{m}^{2}\right)$, mean $\pm \mathrm{DS}$ & $51.83 \pm 12.24$ & $48.02 \pm 14.72$ & $0.341 * *$ \\
\hline $\begin{array}{l}\text { LAVI after } 12 \text { mon. }\left(\mathrm{ml} / \mathrm{m}^{2}\right) \text {, } \\
\text { mean } \pm \text { DS }\end{array}$ & $38.48 \pm 9.41$ & $52.83 \pm 20.83$ & $0.004 * *$ \\
\hline MR degree, n (\%) & & & $0.467 *$ \\
\hline I & $5(21.7)$ & $6(25.0)$ & \\
\hline II & $15(65.2)$ & $12(50.0)$ & \\
\hline III & $3(13.0)$ & $4(16.7)$ & \\
\hline MR degree after 12 mon., $n(\%)$ & & & $0.027^{*}$ \\
\hline I & $16(69.6)$ & $8(33.3)$ & \\
\hline II & $7(30.4)$ & $10(41.7)$ & \\
\hline III & 0 & $3(12.7)$ & \\
\hline $\mathrm{EF}<30 \%, \mathrm{n}(\%)$ & $19(82.6)$ & $18(75.0)$ & $0.524 *$ \\
\hline $\operatorname{IVMD}(\mathrm{ms})$, mean $\pm \mathrm{DS}$ & $50.48 \pm 22.39$ & $32.43 \pm 16.21$ & $0.003 * *$ \\
\hline $\begin{array}{l}\text { IVMD after } 12 \text { mon. (ms), } \\
\text { mean } \pm \text { DS }\end{array}$ & $19.61 \pm 16.30$ & $11.38 \pm 8.03$ & $0.032 * *$ \\
\hline
\end{tabular}

* Chi-square and ** Independent samplest-test were used in data calculations. Group $\mathrm{A}-\Delta \mathrm{EF}$ improvement $\geq 10 \%$, group $\mathrm{B}-\Delta \mathrm{EF}$ improvement $<10 \%$, $\mathrm{EF}$ - ejection fraction, $\triangle \mathrm{EF}$ - changes in ejection fraction, IVMD - interventricular mechanic dyssynchrony, EDD - left ventricular end diastolic diameter, ESD - left ventricular end systolic diameter, LAVI - left atrial volume index, MR - mitral regurgitation. 
Table 2.7

Echocardiogram data with $\Delta E F \geq 10 \%$ and $\Delta E F<10 \% 24$ months after CRT implantation

\begin{tabular}{|c|c|c|c|}
\hline Parameter & $\begin{array}{c}\text { Group A } \\
(\mathbf{n}=\mathbf{2 7})\end{array}$ & $\begin{array}{c}\text { Group B } \\
(\mathrm{n}=18)\end{array}$ & p value \\
\hline MI scars, n (\%) & $7(25.9)$ & $14(77.8)$ & $0.001 *$ \\
\hline \multicolumn{4}{|l|}{ MI scar localisation, n (\%) } \\
\hline Anterior wall & $4(57.1)$ & $3(21.4)$ & \multirow{4}{*}{$0.125^{*}$} \\
\hline Apex & $1(14.3)$ & $4(28.6)$ & \\
\hline Wide anterior wall & $1(14.3)$ & $7(50.0)$ & \\
\hline Posterior wall & $1(14.3)$ & $0(0)$ & \\
\hline $\mathrm{EDD}(\mathrm{mm})$, mean $\pm \mathrm{DS}$ & $70.00 \pm 5.67$ & $68.67 \pm 6.23$ & $0.462 * *$ \\
\hline EDD after 24 mon. $(\mathrm{mm})$, mean \pm DS & $57.18 \pm 12.87$ & $67.89 \pm 7.36$ & $0.003 * *$ \\
\hline $\mathrm{ESD}(\mathrm{mm})$, mean $\pm \mathrm{DS}$ & $59.44 \pm 7.62$ & $55.78 \pm 7.03$ & $0.111 * *$ \\
\hline ESD after 24 mon. (mm), mean \pm DS & $41.99 \pm 11.31$ & $55.33 \pm 11.03$ & $<0.001 * *$ \\
\hline $\operatorname{LAVI}\left(\mathrm{ml} / \mathrm{m}^{2}\right)$, mean \pm DS & $51.59 \pm 13.26$ & $48.14 \pm 14.59$ & $0.415 * *$ \\
\hline LAVI after 24 mon. $\left(\mathrm{ml} / \mathrm{m}^{2}\right)$, mean \pm DS & $37.59 \pm 7.45$ & $53.17 \pm 18.63$ & $0.003 * *$ \\
\hline \multicolumn{4}{|l|}{ MR degree, n (\%) } \\
\hline I & $5(18.5)$ & $5(27.8)$ & \multirow{3}{*}{$0.615^{*}$} \\
\hline II & $16(59.3)$ & $10(55.6)$ & \\
\hline III & $4(14.8)$ & $3(16.7)$ & \\
\hline \multicolumn{4}{|l|}{ MR degree after 24 mon., n (\%) } \\
\hline I & $22(81.5)$ & $5(27.8)$ & \multirow[t]{3}{*}{$0.001^{*}$} \\
\hline II & $3(11.1)$ & $10(55.6)$ & \\
\hline III & 0 & $2(11.1)$ & \\
\hline $\mathrm{EF}<30 \%$ & $22(8 ., 5)$ & $13(7 ., 2)$ & $0.464 *$ \\
\hline $\operatorname{IVMD}(\mathrm{ms})$, mean $\pm \mathrm{DS}$ & $51.37 \pm 19.45$ & $27.65 \pm 13.5$ & $<0.001 * *$ \\
\hline IVMD after 24 mon. (ms), mean \pm DS & $18.74 \pm 21.09$ & $17.56 \pm 10.43$ & $0.804 * *$ \\
\hline
\end{tabular}

* Chi-square and ** Independent samplest-test were used in data calculations. Group $\mathrm{A}-\Delta \mathrm{EF}$ improvement $\geq 10 \%$, group $\mathrm{B}-\Delta \mathrm{EF}$ improvement $<10 \%$, $\mathrm{EF}$ - ejection fraction, $\triangle \mathrm{EF}$ - changes in ejection fraction, IVMD - interventricular mechanic dyssynchrony, EDD - left ventricular end diastolic diameter, ESD - left ventricular end systolic diameter, LAVI - left atrial volume index, MR - mitral regurgitation. 


\subsection{Effect of clinical factors on cardiac resinchronisation therapy}

Clinical parameters did not differ statistically significantly when compared A and B groups before and after CRT implantation (Table 2.8). Analysing effects of total cholesterol on CRT, a statistically significant moderately positive correlation was observed 12 months after CRT implantation $(r=0.375 ; p=0.009)$ and after 24 months $(r=0.326 ; p=0.029 ;)$, which indicated on the better response to CRT treatment in patients without identified statin therapy for coronary heart disease.

Table 2.8

Difference in clinical data in $\triangle E F \geq 10 \%$ and $\Delta E F<10 \%$ groups 24 months after CRT implantation

\begin{tabular}{lccc}
\hline \multicolumn{1}{c}{ Parameter } & $\begin{array}{c}\text { Group A* } \\
(\mathbf{n}=\mathbf{2 7})\end{array}$ & $\begin{array}{c}\text { Group B* } \\
(\mathbf{n}=\mathbf{1 8})\end{array}$ & p value \\
\hline BNP $(\mathrm{pg} / \mathrm{ml})$ & $890.00[650.00 ;$ & $992.50[376.75 ;$ & $0.844^{* * * *}$ \\
& $1541.00]$ & $1870.25]$ & \\
TC $(\mathrm{mmol} / \mathrm{l})$ & $4.99 \pm 1.28$ & $4.17 \pm 1.03$ & $\mathbf{0 . 0 2 9 * *}$ \\
LDLC (mmol/l) & $2.84 \pm 1.08$ & $2.49 \pm 0.91$ & $0.269^{* *}$ \\
HDLC (mmol/l) & $1.22 \pm 0.44$ & $1.09 \pm 0.33$ & $0.301^{* *}$ \\
TG (mmol/l) & $1.40[0.80 ; 1.70]$ & $1.00[0.700 ; 1.40]$ & $0.231^{* * *}$ \\
Hb (g/dl), & $13.84 \pm 1.82$ & $14.10 \pm 1.06$ & $0.556^{* *}$ \\
Glucose (mmol/l) & $6.29 \pm 2.35$ & $6.01 \pm 1.24$ & $0.637^{* *}$ \\
CRO (mg/l) & $2.70[1.65 ; 5.60]$ & $2.1[1.45 ; 5.55]$ & $0.497^{* * *}$ \\
\hline
\end{tabular}

* Median and interquartile interval, ** Independent samples t-test and Mann-Whitney $U$ test were used in data calculations. Group $\mathrm{A}-\Delta \mathrm{EF}$ improvement $\geq 10 \%$, group $\mathrm{B}-\Delta \mathrm{EF}$ improvement $<10 \%, \Delta \mathrm{EF}-$ changes in ejection fraction, $\mathrm{BNP}$ - brain natriuretic peptide, TC - total cholesterol, HDLC - high density lipoprotein cholesterol, LDLC - low density lipoprotein cholesterol, TG - triglycerides, $\mathrm{Hb}$ - haemoglobin, $\mathrm{CRO}-\mathrm{C}$-reactive protein. 


\subsection{Effect of cardiac resinchronisation devices on cardiac resinchronisation therapy}

Localisation of lateral and posterior-lateral electrodes for stimulating left ventricular in coronary sinus was identified in 40 (85.1\%) patients, of which CRT efficacy after 12 months was observed in 21 (52.5\%) compared to other localisations of electrodes -2 (28.6\%); however, statistical significance was not reached $(\mathrm{p}=0.416)$ and 24 months after CRT $-24(63.2 \%)$ vs. $3(42.9 \%$; $\mathrm{p}=0.412 ;$ Chi-square test was used in data calculations).

Left ventricle before right ventricle $(\mathrm{LV} \rightarrow \mathrm{RV})$ was stimulated in 23 (47.9\%) patients. Patients with LV $\rightarrow$ RV pacing statistically significantly more frequently resulted in CRT efficiency group compared to patients with a different pacing sequence, respectively 16 (69.6\%) vs. 7 (30.4\%) 12 months after CRT $(\mathrm{p}=0.006)$ and $17(63.0 \%)$ vs. $10(37.0 \%)$ after 24 months $(\mathrm{p}=0.021)$. Comparison of CRT programmed pacing types have been represented in Figure 2.14.

Effective biventricular pacing ( $\geq 95 \%$ ) was reached by $43(91.5 \%)$ patients 12 months after CRT and 43 (95.6\%) after 24 months. Patients $(n=43)$ with effective biventricular pacing statistically more frequently resulted in CRT efficiency group $23(100 \%)$ compared to patients $(n=4)$ without effective biventricular pacing $0(0 \% ; \mathrm{p}=0.109)$ after 12 months and $27(100 \%)$ vs. 0 $(0 \% ; \mathrm{p}=0.155)$ after 24 months (Chi-square test was used in data calculations). 


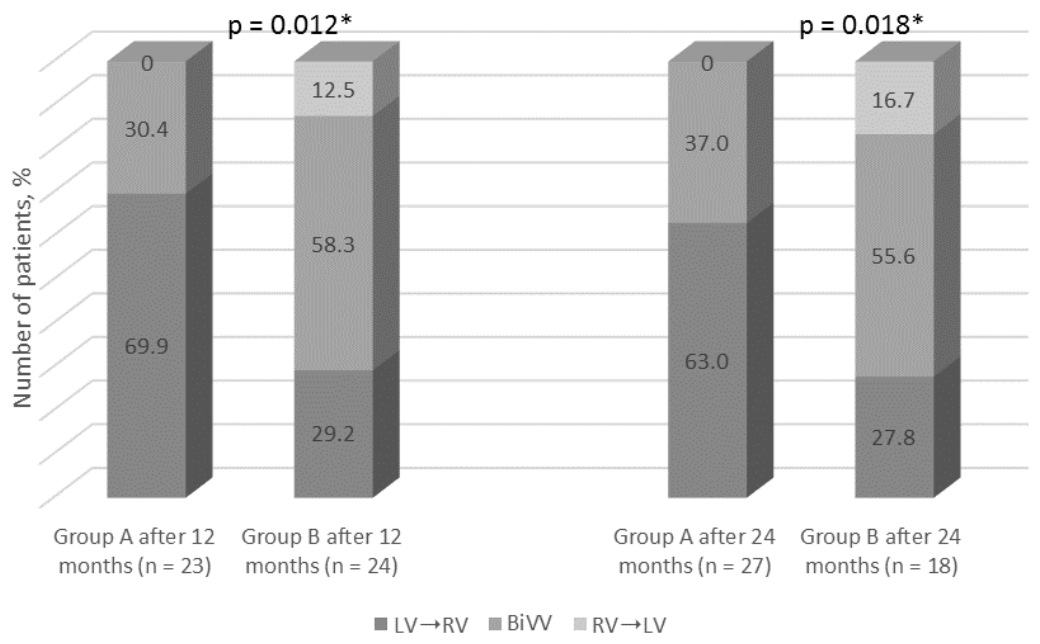

Figure 2.14 Influence of interventricular pacing type on cardiac resinchronisation therapy after 12 and 24 months

* Chi-square test was used in data calculations

Group $\mathrm{A}-\Delta \mathrm{EF}$ improvement $\geq 10 \%$, group $\mathrm{B}-\Delta \mathrm{EF}$ improvement $<10 \%$, $\mathrm{RV} \rightarrow \mathrm{LV}$ - left ventricle before right ventricle, BiVV - biventricular, $\mathrm{RV} \rightarrow \mathrm{LV}$ - right ventricle before left ventricle

\subsection{Prognostic parameters of effective cardiac resinchronisation therapy}

Multivariate analysis (MVA), as prognostic clinical and objective parameter, indicated the impact of LBBB on efficacy of CRT devices after 12 and 24 months, statistically significant IVMD, higher SBP (Tables 2.9 and 2.10). 
Table 2.9

Influencing factors of efficiency of cardiac resinchronisation therapy after 12 months

\begin{tabular}{lcc}
\hline \multicolumn{1}{c}{ Parameter } & Exp B & p value* \\
\hline Gender - female, n (\%) & 0.271 & 0.051 \\
LBBB, n (\%) & 0.283 & 0.056 \\
QRS width, mm & 0.242 & 0.088 \\
\hline
\end{tabular}

*Multiple linear regression backward method was used for data analysis.

LBBB - left His bundle branch block.

Table 2.10

Influencing factors of efficiency of cardiac resinchronisation therapy after 12 months

\begin{tabular}{lcc}
\hline Parameter & Exp B & p value* \\
\hline SBP, mm Hg & 0.235 & 0.040 \\
LBBB, n (\%) & 0.394 & 0.001 \\
IVMD, mm & 0.419 & $<0.001$ \\
\hline
\end{tabular}

* Multiple linear regression backward method was used for data analysis.

SBP - systolic blood pressure, LBBB - left His bundle branch block,

IVMD - interventricular mechanic dyssynchrony. 


\section{DISCUSSION}

Heart failure is a complex clinical syndrome, which occurs due to abnormality of cardiac structure or function leading to worsening of ventricular activity, failure of the heart to provide adequate blood circulation and tissue metabolism. Undoubtedly, the use of pharmacotherapy plays the key role in the treatment of HF; however, there are cases when contractile cardiac functions are limited, and positive effect of the pharmacotherapy cannot be reached. In cases where a contributing factor of HF is electrical dyssynchrony of both ventricles and pharmacological therapy does not give the expected results, the treatment of HF requires an additional use of a cardiac resynchronisation device (CRT). At present, CRT has become a significant component of treatment of heart failure for patients resistant to drug therapy.

This is the first study conducted in Latvia which is carried out with application of CRT for better understanding of the situation in this field in the country and to assess CRT efficacy, as well as to define and evaluate factors predisposing inefficiency of CRT. CRT device implantations in Latvia have been performed since 2006. This study was conducted during the period when more intense rate of implantations of CRT devices was initiated in Latvia (2009 to 2012).

In general, the clinical, longitudinal, prospective, two-arm study included 50 CRT patients with preserved sinus rhythm. All patients underwent CRT device implantation, and the subjective and objective feelings of the patients were evaluated prior to implantation, 12 and 24 months after CRT. Two patients were excluded form the further study, one of which underwent ablation of the radio frequency catheter of the atrioventricular joint, and the other had artificial ventricle implanted. Cardiovascular death due to CHF decompensastion was observed in three patients $-1(2.1 \%)$ patient in three monts and $2(4.2 \%)$ patients 
in 24 months. Therefore, the number of patients included in the study at the 12 months visitation was 47 patients and at the 24 months visitation -45 patients.

Based on the above, after prospective evaluation of EchoCG-measured improvement of the left ventricular ejection fraction $(\mathrm{EF}) \geq 10 \%$, the population of this study was divided into two main groups (group A and B):

- Group A (responders) included patients who 12 and 24 months after a CRT device implantation reached improvement of left ventricular ejection fraction $(\mathrm{EF}) \geq 10 \%$;

- Group B (non-responders) included patients who 12 and 24 months after implantation of a CRT device failed to reach improvement of left ventricular $\mathrm{EF} \geq 10 \%$.

The initial left ventricular ejection fraction $(\mathrm{EF})$ of the study population, according to the EchoCG data, on average was $24.81 \pm 5.23 \%$; after cardiac resynchronisation device implantation, EF statistically significantly increased compared to the intitial EF, respectively by $11.40 \pm 9.31 \%$ after 12 months, $\mathrm{p}<0.001$ and $13.67 \pm 10.84 \%$ after 24 months, $\mathrm{p}<0.001$. High cardiac resynchronisation efficiency $(\Delta \mathrm{EF}$ improvement $\geq 10 \%$ ) after 12 months was observed in $23(48.9 \%)$ patients and after 24 months - $27(60.0 \%)$ patients defined as group A or CRT responders.

It could be assumed that only slightly more than a half of patients achieved EchoCG positive improvement of HF; however. the study showed that CRT efficiency depends not only on EF and positive remodelling of left ventricle. Often improvement of subjective symptoms as well as reduction in hospital admittances was also achieved in the group of patients where EchoCG-measured EF improvement was 5-10\%.

The main task of the study was to analyse differences of groups A and B evaluating 53 different possible factors affecting treatment 12 and 24 months 
after CRT implantation. Correlation analysis was applied for identification of the links between the variables.

\section{Subjective and objective parameters}

The study showed distinct prevalence of male participants $(68.8 \%)$. Nevertheless, compared to males, statistical reliability was observed in females 12 months after CRT, respectively $73.3 \%$ vs. $37.5 \%$; $\mathrm{p}=0.030$, after 24 months a similar trend was maintained, respectively $80.0 \%$ vs. $50.0 \%$, which was close to statistical significance $(p=0.063)$. This leads to the conclusion that following gender-based analysis, females are better candidates for CRT implantation.

Assessment of systolic blood pressure (SBP) on CRT efficiency showed statistically significant moderately strong positive correlation $(\mathrm{r}=0.436$; $\mathrm{p}=0.003$ ) after 24 months, which indicates on a better response to CRT in patients with higher SBP (divide of SBP - $130 \mathrm{~mm} \mathrm{HG).} \mathrm{It} \mathrm{can} \mathrm{be} \mathrm{explained} \mathrm{that}$ lowered SBP may enhance hypoperfusion of vital organs and cause the drop of CRT efficiency.

SBP may not be treated as a prognostic factor to estimate pre-treatment indications by analysing blood pressure figures before CRT implantation. However, in view of indications for CRT implantation, numerical values of blood pressure must be considered, thus ensuring ODT to avoid medication hypotension in patients.

The study did not include active smokers; therefore, this factor was evaluated based on prior length and amount of smoking in the study population. $34(70.8 \%)$ patients were anamnestic smokers. The study concluded that longterm smokers at least 20 cigarettes a day for ten years $(n=34)$ after 12 months

of CRT resulted in CRT responders group less frequently compared to non-smokers $(\mathrm{n}=13)$, respectively $14(41.2 \%)$ vs. $9(69.2 \% ; \mathrm{p}=0.111)$, after 
24 months a similar trend was observed, respectively $17(53.1 \%)$ vs. 10 (76.9\%), although statistical significance was not reached $\mathrm{p}=0.188$.

Assessment of heart failure functional class before CRT implantation showed the dominance of patients with HF III NYHA functional class $70.8 \%$. At 12-months control visit, HF III NYHA was observed in $34.8 \%$ patients and at 24-months control visit - $24.4 \%$ patients. HF II NYHA respectively $14.6 \% ; 60.9 \%$ and $73.3 \%(\mathrm{p}<0.001)$. However, assessment of statistical differences linked to HF functional class, in groups A and B neither after 12 nor 24 months statistically significant correlation was observed $(p=0.466$ after 24 months).

Prior to resinchronisation device implantation, frequent and repeated hospital admissions of patients due to CHF decompensation occurred. However, after CRT device implantation, admissions to hospitals happened less frequently. Respectively, 12 months after CRT four patients were hospitalised once, two patients twice and one patient three times $(\mathrm{p}<0.001)$, and after 24 months - six patients were hospitalised once and two patients twice $(\mathrm{p}<0.001)$. Statistically significant moderate negative correlation was observed between the repeated hospitalisations due to CHF decompensation and CRT efficiency $(r=-0.294$; $\mathrm{p}=0.050$ ), which indicates on a better response to the therapy in patients with fewer hospitalisations before CRT implantation (12 mon. $-\mathrm{r}=-0.259$; $\mathrm{p}=0.078)$.

Patients with re-hospitalisation more than two times due to CHF decompensation before CRT device implantation comparatively less frequently resulted in CRT responders group after 12 months compared to patients with $1-2$ times hospitalisation occurrences, respectively $20.0 \%$ vs. $54.5 \%$, which was close to statistical significance $(\mathrm{p}=0.076)$, a similar trend was observed after 24 months, respectively $30.0 \%$ vs. $67.7 \%$; $=0.063$. 
Evaluating HF etiology, the study patients are typically divided into two large groups - ischemic genesis HF patients, heart failure factor of which is coronary heart disease (CHD) and non-ischemic genesis HF patients, where dilated cardiomyopathy (CMP) is not connected with CHD.

Clinical efficacy of CRT in both groups was assessed in subanalysis of MADIT-CRT trial (Zareba et al., 2011; Barsheshet et al., 2011). The study included 1046 patients with ischemic CMP and 774 patients with non-ischemic CMP, the follow-up took 2.4 years. Patients with ischemic CMP reached $34 \%$ reduction of mortality and clinical deterioration of heart failure if compared CRT-D group with the group of patients with ICD, while patients with non-ischemic CMP reached $44 \%$ reduction of mortality or deterioration of heart failure in CRT-D group compared to the ICD group.

HF development causes of the current study varied; however, ischemic genesis CMP were observed in $28(58.3 \%)$ patients and non-ischemic genesis CPM in 20 (41.7\%) patients. Longitudinal (24 months) study data indicate that statistically significant frequency of CRT efficiency was observed in nonischemic genesis CPM patients (66.7\%) compared to ischemic genesis CMP patients $(44.0 \% ; \mathrm{p}=0.018)$.

Multi-centre trials and this study show that patients with non-ischemic origin of HF benefit more from CRT when compared to patients with ischemic genesis HF. This could be explained by the anatomical structure of left ventricle. In patients after myocardial infarction, HF development mechanism involves hypokinesic or akinesic scars that maintain the HF progression mechanism, while in patients with HF of non-ischemic genesis ventricles are diffusely enlarged and better submit to ventricular pacing, providing smoother conduction of impulses into ventricles.

Thus, based on HF genesis and etiological factors of HF development, it can be concluded that patients having suffered heart attack are poorer responders 
to CRT efficiency. Comparing data of the study in groups A and B, myocardial revascularisation has been traced if such treatment has been conducted before CRT implantation. Patients without identified revascularisation 24 months after CRT statistically significantly more frequently resulted in CRT responders group compared to patients with performed revascularisation, respectively 16 (80.0\%) vs. $11(42.3 \%), p=0.030$. The changes can be linked to CMP genesis, and thus claimed that patients with ischemic CMP have a bigger chance of ending up in CRT non-responders group (B) and patients with non-ischemic genesis in responders group (A).

Statistically significant influence between types of revascularisation and cardiac resinchronisation therapy was not observed.

Before CRT implantation, all patients were ensured OMT. It was limited to several patients due to hypotention, bradycardia, significant kidney damage. CRT efficiency did not differ statistically significantly 12 and 24 months after CRT in patients who received optimal medication therapy (ACEI/ARB, BAB, un ARB) in maximally tolerable doses, according to ESC CHF Guidelines, compared to patients who did not tolerate optimal medication therapy.

Differences in groups A and B were observed in dyslipidemia therapy group. Patients $(n=12)$ without identified statin therapy 24 months after CRT showed a more frequent prevalence resulting in CRT responders group compared to patients $(\mathrm{n}=33)$ who used statins, respectively $10(83.3 \%$ vs. $17(51.5 \%)$, which was close to statistical significance $p=0.086$. This again proved that patients with ischemic CMP, identified with statins after myocardial infarction, more frequently resulted in the non-responders group.

\section{ECG visualisation parameters}

CRT is an effective method in reduction of clinical symptoms of HF in patients with heart failure and widened QRS interval. Studies and meta-analysis 
show that patients with significantly widened QRS interval derive the greatest benefits from CRT (Sipahi et al., 2011; Stavrakis et al., 2012).

The width of QRS complex before CRT implantation for the study patients was $169.71 \pm 22.54 \mathrm{~ms}$. Immediately after CRT implantation the mean width of QRS decreased significantly by $-15.50 \pm 21.12 \mathrm{~ms} ; \mathrm{p}<0.001 .12$ months after CRT, the mean QRS width decreased statistically significantly by $-18.75 \pm 24.71 \mathrm{~ms} ; \mathrm{p}<0.001$; however, after 24 months by $-17.31 \pm 22.34 \mathrm{~ms}$; $\mathrm{p}<0.001$. Assessing the impact of initial mean QRS width on CRT efficiency, a statistically significant moderate positive correlation was observed 12 months after CRT $(r=0.322 ; p=0.027)$ and after $24(r=0.297 ; p=0.048)$, which indicates on a better response to CRT therapy in patients with a wider QRS.

After CRT implantation, statistically significant QRS width correlation with $\Delta \mathrm{EF} \geq 10 \%$ improvement was not observed.

At the beginning of CRT "era", width of QRS complex was considered as one of leading criteria of CRT efficacy, but over the years morphology of QRS complex has also been analysed as a factor contributing to the efficiency. In the MADIT-CRT ( $\mathrm{n}=1820$ ) (Moss et al., 2009; Zareba et al., 2011) study, reduction of mortality and hospital admittances in CRT-D group compared to ICD group was $34 \%$, while in the subgroup which consisted of patients with LBBB $-57 \%$. More pronounced efficacy of CRT, which established long-term efficacy of CRT, is MADIT-CRT (published in 2014) 7-year data analysis of HF patients with moderate course of the disease and markedly reduced systolic left ventricular dysfunction. In the patient group with $\mathrm{LBBB}$, all types of mortality in the CRT-D group was $18 \%$ compared to the ICD group, where mortality over the 7-year observation was $29 \%$. This suggests that nine HF patients should be treated with CRT to save one life in the 7-year period (Goldenberg et al., 2014).

In the current study, there were 37 (77.1\%) patients with LBBB before CRT implantation. 12 months after CRT, patients with LBBB $(n=36)$ showed 
CRT efficiency statistically significantly more frequently compared to patients with $\operatorname{RBBB}(\mathrm{n}=9)$ or without EchoCG dyssynchrony without wide QRS $(\mathrm{n}=2)$, respectively $22(61.1 \%)$ vs. $1(11.1 \%)$ vs. $0(0 \%$; $=0.030), 24$ months after CRT a similar trend was maintained, respectively 26 (74.3\%) vs. $1(12.5 \%)$ vs. $0(0 \% ; p=0.001)$. Assessment of correlation proved that moderately close link exists between improvement and QRS complex morphology. The longer the period since patient randomisation in the study, the better improvement for the patients with LBBB.

Left His bundle branch full block is a decisive criterion of CRT efficiency. This could be explained by more pronounced adverse remodelling of left ventricle in this patient population. Consequently, the CRT therapy may give greater improvement. This is shown in international studies, and a convincing confirmation is also this study carried out in Latvia. Research data also confirm improvement of lower HF functional class in patients with RBBB (Leong et al., 2012) which could probably correlate with inferior CRT efficiency.

\section{EchoCG parameters}

Numerous international studies (Daubert et. al., 2009; Zareba et. al., 2011; Tang et. al., 2010) compare patients with identified scars in left ventricle with patients with much rarer EchoCG identified scars or possessing diffuse hypokinesia of left ventricle.

The study included $24(50.0 \%)$ patients with post-infarction scar, according to EchoCG data, $13(27.1 \%)$ patients possessing diffuse hypokinesia of left ventricle; however, 11 (22.9\%) patients had no disturbed myocardial contractility. Patients $(n=23)$ detected with a scar in one of the walls of left ventricle statistically less frequently resulted in CRT efficiency group; 6 $(26.1 \%)$ compared to patients $(\mathrm{n}=24)$ without post-infarction scar $17(73.9 \%$; $\mathrm{p}=0.002) 12$ months after CRT and $7(25.9 \%)$ vs. $20(74.1 \%$; $=0.001)$ after 
24 months. The groups differed, and the data, similarly to HF CMP genesis, indicated on HF patients with ischemic genesis as weaker candidates to CRT efficiency.

The study revealed statistically significant decrease in size of cavity of left ventricle after CRT (EDD by $-4.89 \pm 11.60 \mathrm{~mm}$; $\mathrm{p}=0.006$ after 12 months and $-8.00 \pm 13.16 \mathrm{~mm} ; \mathrm{p}<0.001$ after 24 months; ESD by $-8.47 \pm 13.01 \mathrm{~mm}$; $\mathrm{p}<0.001$ and $-10.65 \pm 14.19 \mathrm{~mm} ; \mathrm{p}<0.001 ;$ LAVI by $-4.07 \pm 18.51 \mathrm{~mm}$; $\mathrm{p}=0.138$ and $-6.39 \pm 15.78 \mathrm{~mm} ; \mathrm{p}=0.009)$. Statistically significant decrease was observed in IVMD 12 months after CRT; by $-26.33 \pm 21.26 \mathrm{~ms} ; \mathrm{p}<0.001$ and by $-23.84 \pm 24.33 \mathrm{~ms} ; \mathrm{p}<0.001$. Moreover, after CRT implantation statistically significant improvement was observed in degree of patient mitral regurgitation, respectively upon hospitalisation degree III was observed in 7 (14.6\%), after 12 months - 3 (6.4\%), 24 months later - 2 (4.4\%) patients; $\mathrm{p}<0.001$.

Assessing initial IVMD impact on CRT efficiency, statistically significant moderate positive correlation was observed after 12 months $(r=0.427 \mathrm{p}=0.003)$ and strong correlation after 24 months $(\mathrm{r}=0.560, \mathrm{p}<0.001)$, which indicates on a better response to CRT in patients with a more distinct IVMD before CRT implantation.

\section{Most significant clinical parameters}

One of the objectives of the research was to trace HF biomarker BMP changes while in action. After CRT implantation, mean BNP median statistically significantly decreased form $939.00 \mathrm{pg} / \mathrm{ml}[517.50 ; 1769.25]$ to $320.00 \mathrm{pg} / \mathrm{ml}$ [148.00; 653.00] after 12 months $(\mathrm{p}<0.001)$ and $189.00 \mathrm{pg} / \mathrm{ml}[100.50 ; 518.50]$ after 24 months $(\mathrm{p}<0.001)$. The data, similarly to the data by EchoCG on heart cavity decrease, indicate on longitudinal positive CRT efficiency. 
Evaluating total cholesterol impact on CRT efficacy, statistically significant moderate positive correlation was observed both 12 months after CRT $(\mathrm{r}=0.375 ; \mathrm{p}=0.009)$, and 24 months after CRT $(\mathrm{r}=0.326 ; \mathrm{p}=0.029)$, which indicates on a better response to CRT in patients without identified statin therapy due to coronary heart desease.

\section{Ventricular dyssynchrony programmed parameters for CRT device}

Contemporary CRT devices allow very variable programming of right and left ventricle contraction time, and it is labelled as VV interval. The ventricular pacing parameters are programmed into CRT device to prevent dyssynchrony, according to CRT ventricular VV dyssynchrony severity and direction (which ventricle contracts first). The study patients with programmed left ventricle pacing before the right one statistically significantly more frequently resulted in CRT efficiency group compared to patients with a different pacing sequence, respectively 16 (69.6\%) vs. 7 (30.4\%) 12 months after CRT $(\mathrm{p}=0.006)$ and $17(63.0 \%)$ vs. $10(37.0 \%)$ after 24 months $(\mathrm{p}=0.021)$.

Therefore, it can be concluded that patients with LBBB, whose right ventricle contracts first and the left ventricles contraction is delayed, and CRT mode is LV $\rightarrow \mathrm{RV}$ can be considered as better candidates for effective CRT.

The study analyses ratio of pacing of ventricles, by optimising atrioventricular (AV) and interventricular (VV) conduction during the process of CRT device programming, by assessing differences in CRT responders and nonresponders groups.

CRT efficiency is based on close to $100 \%$ provision of pacing of both ventricles. Percentage decrease of biventricular pacing causes decrease in positive efficiency of resinchronisation therapy. To prevent such ocurrence, patients were carefully monitored during the study, adjusting both ventricular pacing parameters of CRT and assessing the need for pharmacological treatment 
of tachyarrhythmia due to frequent extrasystoles or atrial fibrillation. One patient was excluded from further analysis of the study and underwent atrioventricular connection catheter ablation due to constantly recurrent, medically refractory atrial fibrillation. In this study, all patients were provided the best possible biventricular pacing to achieve better efficiency of CRT. Biventricular pacing was lower than $95 \%$ in only 4 patients and all these patients ended up in the non-respionders group of the therapy.

One of the objectives of the research was to assess localisation of left ventricular electrode of a CRT device in coronary sinus branches, radiographically evaluating correlation between positioning of an electrode and efficiency of CRT.

International, multi-centre studies show that localisation of an electrode for left ventricular pacing in the coronary sinus is important in ensuring effectiveness of CRT. At present, lateral and posterolateral CS vein is regarded as the best site for localisation of the electrode, while the results of anterior and apical pacing of CS vein are worse. The analysis of MADIT-CRT study compared position of CS apical pacing with pacing positions of the rest of the left ventricle. Apical left ventricular pacing is associated with poorer efficiency of CRT (Singh et al., 2011).

In this, first time in Latvia, study targeted at CRT efficacy, it was important to assess sites of localisation of left ventricular electrodes, because it is one of the most important criteria for positive efficiency of CRT. Coronary sinus localisation of an electrode in lateral wall was identified in $45.8 \%$ patients, posterolateral wall in $39.6 \%$, anterolateral wall in $6.3 \%$ and posterior wall in $8.3 \%$ patients. Localisation of left ventricular electrodes in posterior and posterolateral wall were identified in $40(85.1 \%)$ patients, of which CRT efficacy after 12 months was observed in 21 (52.5\%) compared to other localisations of electrodes $2(28.6 \%)$; however, statistical significance was not 
reached $(\mathrm{p}=0.416)$ and after 24 months $24(63.2 \%)$ vs. $3(42.9 \% ; \mathrm{p}=0.412)$. Thus, it can be concluded that in case of 7 patients, localisation of CS electrode for pacing of left ventricle was not the most favourable positioning, and five of the patients belonged to the group of patients with $\mathrm{EF} \geq 10 \%$ improvement reached after 12 months. 


\section{CONCLUSIONS}

1. High CRT efficiency within two years was reached by nearly two thirds of the study population with the total growth of left ventricle ejection fraction by approximately $14 \%$ p.

2. CRT implantation is a safe and efficient HF treatment method. CRT statistically significantly improves patient life quality by significantly reducing the amount of hospital admissions, improving cardio load tolerance and decreasing remodelling of left ventricle. Device-related complications or adverse cardiovascular events were not observed during the study.

3. Patients with non-ischemic cardiomyopathy, systolic blood pressure above $130 \mathrm{~mm} \mathrm{Hg}$ and those with fewer hospital admissions due to $\mathrm{HF}$ decompensation showed statistically significantly larger CRT efficiency over a longer period of time.

4. Patients with a wider QRS complex (>170 ms) and morphologic left His bundle branch block before CRT implantation were more responsive to CRT.

5. Patients with post-infarction scar, according to EchoCG data, showed poorer CRT results compared to patients with diffuse hypokinesia or without disturbed myocardial contractility. Patients with significantly larger interventricular mechanic dyssynchrony $(>50 \mathrm{~ms})$ before CRT implantation reached a better longitudinal CRT efficiency. Impact of clinical indicators on CRT efficiency was not observed. 
6. Patients with programmed left ventricle pacing before the right one statistically significantly more frequently reached higher CRT efficiency compared to patients with a different pacing sequence. Impact of other device-related factors on CRT efficiency was not observed.

7. Dominant parameters affecting longitudinal CRT efficiency are initially LBBB, interventricular mechanic dyssynchrony $>50 \mathrm{~ms}$ and SBP above $130 \mathrm{~mm}$ HG. 


\section{PRACTICAL RECOMMENDATIONS}

Patient selection and objective assessment of implantation criteria before CRT implantation is significant in the further course of disease and clinical outcomes. CRT implantation criteria is a set of objectivisable data, which may provide data on the prospective efficiency of the implantable device.

The created recommended CRT prospective efficiency and patient selection table may serve as a practical tool for selecting patients for cardiac resinchronisation device implantations, as well as prognosticating their performance efficiency (Table 5.1).

Table 5.1

CRT prospective efficiency patient selection

\begin{tabular}{|c|c|c|c|}
\hline $\begin{array}{l}\text { CRT efficiency } \\
\text { determining factor }\end{array}$ & $\begin{array}{l}\text { Good candidates } \\
\text { for CRT }\end{array}$ & $\begin{array}{c}\text { Moderate } \\
\text { candidates for } \\
\text { CRT }\end{array}$ & Poor candidates for CRT \\
\hline QRS width & $\geq 150 \mathrm{~ms}$ & $\leftarrow<>\rightarrow$ & $<120 \mathrm{~ms}$ \\
\hline QRS morphology & LBBB & $\leftarrow<>\rightarrow$ & $\begin{array}{l}\text { LBBB or interventricular } \\
\text { mechanic dyssynchrony } \\
\text { without wide QRS }\end{array}$ \\
\hline $\begin{array}{l}\text { Left } \\
\text { cavities }\end{array}$ & Bigger & $\leftarrow<>\rightarrow$ & Smaller \\
\hline $\begin{array}{l}\text { Ventricular } \\
\text { dyssynchrony }\end{array}$ & Yes & $\leftarrow<>\rightarrow$ & No \\
\hline $\begin{array}{l}\text { Localisations of } \mathrm{CS} \\
\text { electrode }\end{array}$ & $\begin{array}{l}\text { Lateral and } \\
\text { posterolateral CS } \\
\text { vein }\end{array}$ & $\leftarrow<>\rightarrow$ & Anterior and apical CS vein \\
\hline Gender & Female & $\leftarrow<>\rightarrow$ & Male \\
\hline $\begin{array}{ll}\text { Systolic } & \text { Blood } \\
\text { Pressure } & \end{array}$ & Above $130 \mathrm{~mm} \mathrm{Hg}$ & $\leftarrow<>\rightarrow$ & Below $130 \mathrm{~mm} \mathrm{HG}$ \\
\hline $\begin{array}{l}\text { Functional class of } \\
\text { heart failure }\end{array}$ & NYHA II & $\leftarrow<>\rightarrow$ & NYHA IV \\
\hline Genesis of heart failure & Non-ischemic & $\leftarrow<>\rightarrow$ & Ischemic \\
\hline $\begin{array}{l}\text { Ratio of pacing of } \\
\text { boths ventricles }\end{array}$ & $\begin{array}{l}\text { Close to } 100 \% \\
\text { biventricular pacing }\end{array}$ & $\leftarrow<$ & $\begin{array}{l}\text { Less than } 90 \% \\
\text { biventricular pacing }\end{array}$ \\
\hline Co-existent illnesses & No & $\leftarrow<>\rightarrow$ & Yes \\
\hline Re-hospitalisation & Rarer & $\leftarrow<>\rightarrow$ & More frequent \\
\hline
\end{tabular}

LBBB - left His bundle branch block, RBBB - right His bundle branch block, CS - coronary sinus, NYHA - New York Heart Association. 


\section{Articles on the subject of the study}

\section{Publications (scientific articles) on the subject of the study}

1. Vikmane, M., Kalejs, O., Kamzola, G., Upite, D. Ventina, M., Nesterovics, N., Lejnieks, A. Assessment of cardiac resynchronisation therapy efficacy determining factors for patients with moderate and severe heart failure in the population of Latvia in a 12 and 24 month study. Proceeding of the Latvian Academy of Sciences. Section $B$, Vol. 72 (2018). No.6 (717), pp. 20-30.

2. Strēlnieks, A., Bērziņš, A., Karakone, M., Pupkeviča, I., Jubele, K., Vikmane, M., Sakne, S., Kalējs, O., Lejnieks, A. Lejnieks. Comparison of effectiveness and safety of antiarrhytmic drugs class IC and III in patients after electrical cardioversion. Proceedings of Latvian Academy of Science, 2018. Proceeding of the Latvian Academy of Sciences. Section B, Vol. 73 (2019), No. 1 (718), 34-39.

3. Bänsch, D., Bonnemeier, H., Brandt, J. et. al. Collaborator Vikmane, M. et al. Intraoperative defibrillation testing and clinical shock efficacy in patients with implantable cardioverter-defibrillators: the NORDIC ICD randomized clinical trial. Eur Heart J. 2015 Oct 1; 36 (37): 2500-2507.

4. Vikmane, M., Kalējs, O., Kamzola, G., Zabunova, M., Pudulis, J., Vanaǵele, D., Lejnieks, A. Sirds resinhronizācijas iekārtu implantācijas efektivitātes kritēriju izvērtējums pacientu ārstēšanā ar mērenu un smagu sirds mazspēju Latvijas populācijā (Eng. Assessment of cardiac resinchronisation device implantation efficiency in treatment of patients with moderate and severe heart failure in Latvian population). RSU Zinātniskie raksti. 2014, 157-171.

5. Vikmane, M., Kalējs, O., Semjonova, O., Lejnieks, A., Kamzola, G., Zabunova, M. Subjektīvu un objektīvu diagnostisko parametru korelācija pacientiem 12 mēnešus pēc sirds resinhronizācijas terapijas (Eng. Correlation of subjective and objective diagnostic parameters in patients 12 months after cardiac resinchronisation therapy). RSU Zinātniskie raksti. 2013, 116-127.

6. Nesterovičs, N., Kalējs O., Blumbergs, M., Vikmane, M., Kamzola, G., Zabunova, M., Strēlnieks, A., Lejnieks, A. Dažādu sirds kambaru elektrostimulācijas metožu salīdzinošā efektivitāte pacientiem ar sirds mazspēju un pastāvīgu ātriju fibrilāciju (Eng. Compative efficiency of various ventricle electropacing methods in patients with heart failure and permanent atrial fibrillation). RSU Zinātniskie Raksti 1. sējums. 2010, 146-164.

7. Kalejs, O., Nesterovics, N., Blumbergs, M., Ansabergs, J., Sakne, S., Stabulniece, M., Vikmane, M., Lejnieks, A. Effectiveness of Radiofrequency catheter Ablation on Atrioventricular Junction in Patients with permanent Atrial Fibrillation and Heart Failure: Results of Follow - up 72 months. Collections of Scientific Papers, Riga Stradins University. 2009, (5-17).

\section{Published conference abstracts on the subject of the study}

1. Kalejs, O., Jubele, K., Vikmane, M., Sipacevs, P., Nesterovics, N., Lejnieks, A. Intrahospital arrhythmias - who are we now and what we can doing? Latvian Academy of sciences. 2008, 62 (4/5), 71-76. 
2. Kalejs, O., Nesterovics, N., Kamzola, G., Blumbergs, M., Stabulniece, M., Jubele, K., Vikmane, M., Ansabergs, J., Erglis, A. The comparative effectiveness of different pacing methods for patients with heart failure and atrial fibrillation after radiofrequency catheter ablation. Cardiology. 2009, 113, 67-68.

3. Nesterovičs, N., Kalejs, O., Kamzola, G., Blumbergs, M., Stabulniece, M., Jubele, K., Sipacovs, P., Vikmane, M., Erglis, A. The comparative effectiveness of different pacing methods for patients with heart failure and atrial fibrillation after radiofrequency catheter ablation. Journal of Cardiovascular Electrophysiology. 2009, 20 (1), 47-48.

4. Vikmane, M., Kalejs, O., Nesterovics, N., Kamzola, G., Lejnieks, A. Comparison of different efficiency parameters in patients with permanent atrial fibrillation and cardiac resynchronization therapy after catheter ablation. Journal of Cardiovascular Electrophysiology. 2011, 22 (1), 173-182.

5. Vikmane, M., Kamzola, G., Ansabergs, J., Nesterovics, N., Blumbergs, M., Zabunova, M., Kalejs, O., Erglis, A. Benefit from CRT is Similar in Patients with Insignificant and Without Coronary Artery Disease. Journal of Cardiovascular Electrophysiology. 2012, 20 (1), 24-32.

6. Vikmane, M., Semjonova, O., Kamzola, G., Stabulniece, M., Balcere, K., Strelnikova, K., Kalejs, O., Lejnieks, A., Erglis, A. Subjective and Objective Diagnostic Parameters in Patient 12 Months After Cardiac Resynchronisation Therapy. Cardiology. 2013, 1 (9), 40.

\section{Reports in international congresses, conferences}

1. Vikmane, M., Semjonova, O., Kalejs, O., Lejnieks, A. Subjective and objective diagnostic parameter correlation in patients 12 months after cardiac resynhronization therapy. The Heart Failure Association of the European Society of Cardiology. The Heart Failure Congress 2013 Lisbon, Portugal. May 25 to 28, 2013 (poster report).

2. Kalejs, O., Vikmane, M., Kamzola, G., Berezina, M., Zabunova, M., Sakne, S., Nesterovics, N., Lejnieks, A., Erglis, A. The comparatve effectiveness of different pacing sites during radiofrequency catheter ablation for patients with heart failure and atrial fibrillation. 34th Annual Scientific Session. Heart Rhythm 2013, May 8-11, 2013. Denver, Colorado, USA (poster report).

3. Vikmane, M., Semjonova, O., Kamzola, G., Stabulniece, M., Balcere, K., Strelnikova, K., Kalejs, O., Lejnieks, A., Erglis, A. Subjective and Objective Diagnostic Parameters in Patient 12 Months After Cardiac Resynchronisation Therapy. XXIV Nordic Baltic Congress of Cardiology 2013, Oslo Norway. June 1315, 2013. (poster report).

4. Vikmane, M., Kalējs, O., Nesterovics, N. The comparative effectiveness of different pacing sites during radiofrequency catheter ablation for patients with heart failure and atrial fibrilation. The $9^{\text {th }}$ Annual Scientific Congress of the European Cardiac Arrhythmia Society "ECAS” Paris, France April 14 to 16, 2013 (oral report).

5. Vikmane, M., Kalejs, O., Kamzola, G., Ansabergs, J., Nesterovics, N., Blumbergs, M., Zabunova, M., Erglis, A. Benefit from CRT is Similar in Patients with Insignificant and Without Coronary Artery Disease. ECAS 2012: $8^{\text {th }}$ Annual Congress 
of European Cardiac Arrhythmia Society. Munich, Germany. April 20-22, 2012 (poster report).

6. Vikmane, M., Kamzola, G., Nesterovics, N., Blumbergs, M., Ansabergs, J., Zabunova, M., Kalejs, O., Erglis, A. Comparison of different efficiency parameters in patients with permanent atrial fibrillation and cardiac resynchronization. Arrhythmia Lithuania 2012. Vilnas, Lithuania. September 28-29, 2012 (poster report).

7. Kalejs, O., Vikmane, M., Kamzola, G., Ansabergs, J., Nesterovics, N., Blumbergs, M., Zabunova, M., Erglis, A. Benefit from CRT patiens with Coronary artery disease. Abstract. The 11th International Dead Sea Symposium on cardiac Arrhythmias and Device Therapy. Jerusalem. February 26-29, 2012 (poster report).

8. Vikmane, M., Kalejs, O., Nesterovics, N., Kamzola, G., Lejnieks, A. Comparison of different efficiency parameters in patients with permanent atrial fibrillation and cardiac resynchronization therapy after catheter ablation. Venice Arrhythmias 2011. Session: AF electrical therapy. 9 October 2011 (oral report).

9. Vikmane, M. Management of surgical patient with pacemaker. Riga Stradins University. Ziņojums Latvijas anesteziologu un reanimatologu asociācijas organizētos tālākizglìtības kvalifikācijas apmācībā pēc Eiropas Anesteziologijas Izglītības Komitejas programmas. 2011. gada 13. septembris (oral report).

\section{Reports in congresses and conferences in Latvia}

1. Vikmane, M., Ansaberga, I., Kalējs, O., Kamzola, G., Lejnieks, A. Terapijas efektivitātes predisponējošo faktoru izvērtējums mērenas un smagas, hroniskas sirds mazspējas ārstēšanā, pielietojot sirds resinhronizācijas iekārtas. RSU 2014. gada Zinātniskā konference (oral report).

2. Vikmane, M., Semjonova, O., Kamzola G., N̦esterovičs, N., Kalējs, O., Lejnieks, A..Sirds resinhronizācijas terapijas ietekme uz pacientu subjektīvu un objektīvu diagnostisko parametru izmaiņām 12 mēnešus pēc ierīces implantācijas. RSU 2013. gada Zinātniskā konference (oral report).

3. Kalējs, O., Vikmane, M., Nesterovičs, N., Kamzola, G., Zabunova, M., Jubele, K., Lejnieks, A. Dažādas efektivitātes kritēriju salīdzinoša informativitāte pacientiem ar àtriju mirgošanu pēc katetrablācijas un sirds resinhronizācijas. 2012. gada RSU Zinātniskā konference (oral report).

4. Vikmane, M., Kamzola, G., Glāzere, L., Ansaberga, I., Mečniks, P., Nesterovičs, N., Blumbergs, M., Ansabergs, J., Jubele, K., Kalējs, O., Lejnieks, A. Sirds resinhrozizācijas terapijas salīdzinājums sirds mazspējas pacientiem ar išēmiskas un neišēmiskas ǵenēzes kardiomiopātijām. 2012. gada RSU Zinātniskā konference (oral report). 


\section{Bibliography}

1. ACC/AHA/NASPE 2002 Guideline Update for Implantation of Cardiac Pacemakers and Antiarrhythmic Devices: Summary Article. Circulation. 2002, 106, 2145-2161.

2. Arribas, F., Auricchio, A., Boriani, G., et al. Statistics on the use of cardiac electroic devices and electrophysiological procedures in 55 ESC countries: 2013 report from the European Heart Rhythm Association (EHRA). Europace. 2014, 16, i1-i78.

3. Barsheshet, A., Goldenberg, I., Moss, A. J., Eldar, M, Huang, D. T., Mc Nitt, S., Klein, H. U., Jackson, Hall, W., Brown, M. W., Goldberger, J. J., Goldstein, R. E., Schuger, C., Zareba, W. and Daubert, J. P. Response to preventive cardiac resynchronization therapy in patients with ischaemic and nonischaemic cardiomyopathy in MADITCRT. European Heart Journal. 2011, 32, 1622-1630.

4. Daubert, J. C., Saxon, L., Adamson, P. B., Auricchio, A., et. al. 2012 EHRA/HRS expert consensus statement on cardiac resynchronization therapy in heart failure: implant and follow-up recommendations and management. Europace. 2012, 14 (9), 1236-1286.

5. Daubert, C., Gold, M. R., Abraham, W. T.,Ghio, S., Hassager, C., Goode, G., SziliTörök, T., Linde, C. REVERSE Study Group. Prevention of disease progression by cardiac resynchronization therapy in patients with asymptomatic or mildly symptomatic left ventricular dysfunction: insights from the European cohort of the REVERSE (Resynchronization Reverses Remodeling in Left Ventricular Dysfunction) trial. J Am Coll Cardiol. 2009, 54, 1837-1846.

6. Dickstein, K., Cohen-Solal, A., Filippatos, G., Mc Murray, J. J., Ponikowski, P., Poole-Wilson, P. A., Strömberg, A., van Veldhuisen, D. J., Atar, D., Hoes, A. W., Keren, A., Mebazaa, A., Nieminen, M., Priori, S. G. and Swedberg, K. ESC Committee for Practice Guidelines (CPG). ESC Guidelines for the diagnosis and treatment of acute and chronic heart failure 2008: the Task Force for the Diagnosis and Treatment of Acute and Chronic Heart Failure 2008 of the European Society of Cardiology. Developed in collaboration with the Heart Failure Association of the ESC (HFA) and endorsed by the European Society of Intensive Care Medicine (ESICM). Eur Heart J. 2008, 29 (19), 2388-2442.

7. Dickstein, K., Vardas, P. E., Auricchio, A., Claude, J., et al. Guidelines: 2010 Focused Update of ESC Guidelines on device therapy in heart failure: An update of the 2008 ESC Guidelines for the diagnosis and treatment of acute and chronic heart failure and the 2007 ESC guidelines for cardiac and resynchronization therapy Developed with the special contribution of the Heart Failure Association and the European Heart Rhythm Association. Eur Heart J. 2010, 31 (21), 2677-2687.

8. Goldenberg, I., Kutyifa, V., Klein, H. U., Cannom, D. S., Brown, M. W., Dan, A., Daubert, J. P., et. al. Survival with Cardiac-Resynchronization Therapy in Mild Heart Failure. N Engl J Med. 2014, 370 (18), 1694-701. 
9. Leong, D. P., Höke, U., Delgado, V., Auger, D., Thijssen, J., van Erven, L., Bax, J. J., Schalij, M. J. and Ajmone, Marsan, N. Predictors of long-term benefit of cardiac resynchronization therapy in patients with right bundle branch block. Eur Heart J. 2012, 33 (15), 1934-1941.

10. Mann, D. L. Heart Failure: A Companion to Braunwald's Heart Disease, $2^{\text {nd }}$ ed. Elsevier, 2011, 389-390.

11. McMurray, J. J. V., Adamopoulos, S., Anker, S. D., Auricchio, A., Bohm, M., Dickstein, K., Falk, V., Filippatos, G., Fonseca, C., Sanchez, M. A. G., Jaarsma, T., Køber, L., Lip, G. Y. H., Maggioni, A. P., Parkhomenko, A., Pieske, B. M., Popescu, B. A., Rønnevik, P. K., Rutten, F. H., Schwitter, J., Seferovic, P., Stepinska, J., Trindade, P. T., Voors, A. A., Zannad, F. and Zeiher F. ESC Guidelines for the diagnosis and treatment of acute and chronic heart failure 2012. The Task Force for the Diagnosis and Treatment of Acute and Chronic Heart Failure 2012 of the European Society of Cardiology. Developed in collaboration with the Heart Failure Association (HFA) of the ESC. Eur Heart J. 2012, 33 (14), 1787-1847.

12. Moss, A. J., Hall, W. J., Cannom, D. S., Klein, H., Brown, M. V., Daubert, J. P., Mark Estes, III, N. A., Foster, E., Greenberg, H., Higgins, S. L., Pfeffer, A., Solomon, S. D., Wilber, D., and Zareba, W. for the MADIT-CRT Trial Investigators. CardiacResynchronization Therapy for the Prevention of Heart-Failure Events. N Engl J Med. 2009, 361, 1329-1338.

13. Singh, J. P., Klein, H. U., Huang, D. T., et al. Left ventricular lead position and clinical outcome in the multicenter automatic defibrillator implantation trial-cardiac resynchronization therapy (MADIT-CRT) trial. Circulation. 2011, 123, 1159-1166.

14. Sipahi, I., Carrigan, T. P., Rowland, D. Y., et al. Impact of QRS duration on clinical event reduction with cardiac resynchronization therapy: meta-analysis of randomized controlled trials. Arch Intern Med. 2011, 171, 1454-1462.

15. Stavrakis, S., Lazzara, R. and Thadani, U. The benefit of cardiac resynchronization therapy and QRS duration: a meta-analysis. J Cardiovasc Electrophysiol. 2012, 23, $163-168$.

16. Tang, A. S., Wells, G. A., Talajic, M., Arnold, M. O., Sheldon, R., Connolly, S., Hohnloser, S. H., Nichol, G., Birnie, D. J., Sapp, J. L., Yee, R., Healey, J. S., and Rouleau, J. L. for the Resynchronization-Defibrillation for Ambulatory Heart Failure Trial (RAFT) Investigators. Cardiac-resynchronization therapy for mild-to-moderate heart failure. $N$ Engl J Med. 2010, 363, 2385-2395.

17. Zareba, W., Klein, H., Cygankiewicz, I., Hall, W. J., McNitt, S., Brown, M., Cannom, D., Daubert, J. P., Eldar, M., Gold, M. R., Goldberger, J. J., Goldenberg, I., Lichstein, E., Pitschner, H., Rashtian, M., Solomon, S., Viskin, S., Wang, P. and Moss, A. J. MADIT-CRT Investigatorsets. Effectiveness of resynchronization therapy by QRS morphology in the Multicenter Automatic Defibrillator Implantation Trial-Cardiac Resynchronization Therapy (MADIT-CRT). Circulation. 2011, 123, 1061-1072. 